\title{
RMP Colloquia
}

\section{Universality of ac conduction in disordered solids}

\author{
Jeppe C. Dyre and Thomas B. Schrøder \\ Department of Mathematics and Physics, Roskilde University, DK-4000 Roskilde, Denmark
}

\begin{abstract}
The striking similarity of ac conduction in quite different disordered solids is discussed in terms of experimental results, modeling, and computer simulations. After giving an overview of experiment, a macroscopic and a microscopic model are reviewed. For both models the normalized ac conductivity as a function of a suitably scaled frequency becomes independent of details of the disorder in the extreme disorder limit, i.e., when the local randomly varying mobilities cover many orders of magnitude. The two universal ac conductivities are similar, but not identical; both are examples of unusual non-power-law universalities. It is argued that ac universality reflects an underlying percolation determining dc as well as ac conductivity in the extreme disorder limit. Three analytical approximations to the universal ac conductivities are presented and compared to computer simulations. Finally, model predictions are briefly compared to experiment.
\end{abstract}

\section{CONTENTS}

I. Introduction

II. Preliminaries

III. Ac Conduction in Disordered Solids: Facts

IV. Macroscopic Model
A. Definition

B. Ac universality in the extreme disorder limit

V. Symmetric Hopping Model

A. Definition

B. Ac universality in the extreme disorder limit

VI. Cause of Universality

A. Role of percolation

B. Percolation based approximations

VII. Discussion

A. Model predictions

B. Models versus experiment

C. Outlook

Acknowledgments

References

873

873

874

877

877

878

880

880

882

883

883

885

887

887

888

888

890

890

\section{INTRODUCTION}

Though not generally appreciated, everyday materials like glass or plastic have electrical properties remarkably in common. For example, with few exceptions the dc conductivity is Arrhenius temperature dependent. Our focus here, however, is on the strikingly universal ac properties: It is almost always possible to scale measurements of the frequency-dependent conductivity at different temperatures into one single "master" curve. Different solids have quite similar master curves. In particular, ac electronic and ionic conduction cannot be distinguished. The only common feature of the numerous different solids exhibiting this ac universality is their disorder.

What causes ac universality? This has been an open question ever since the full scope of ac universality was recognized in the 1970s (Isard, 1970; Namikawa, 1975; Jonscher, 1977; Owen, 1977; Mansingh, 1980). Recent experiments on ion conducting glasses by Roling et al.
(1997), Ghosh and Sural (1999), and Sidebottom (1999) have renewed interest in ac universality.

Below we review two models for ac conduction in disordered solids, a macroscopic model and a microscopic model. These models embody Occam's razor ${ }^{1}$ by having essentially just one ingredient: disorder. For both models the ac conductivity is independent of the details of the disorder when the local mobilities cover many orders of magnitude-approaching the extreme disorder limit. The universal ac conductivities of the two models are similar and are both close to experimental results. We argue that for both models ac universality is caused by an underlying percolation dominating conduction at extreme disorder.

Why should nonspecialists care about the subject of this Colloquium? One reason is the ubiquity of disordered materials-most likely there are several solids with universal ac conductivity in the very room in which you sit. A second reason has to do with modeling. In modern condensed-matter physics the extreme disorder limit is not often considered. For ac conduction in disordered solids this limit leads to unusual non-power-law universalities. Similar universalities may very well occur elsewhere in "disordered" physics.

\section{PRELIMINARIES}

If $\mathbf{J}_{\text {tot }}$ is the current density (with contributions from both free and bound charges) and $\mathbf{E}$ is the electric field, the conductivity $\sigma_{\text {tot }}$ is defined by $\mathbf{J}_{\text {tot }}=\sigma_{\text {tot }} \mathbf{E}$. In general the conductivity is frequency dependent: If $\omega$ is the angular frequency $\sigma_{\text {tot }}(\omega)$ is the complex quantity defined by $\mathbf{J}_{\text {tot }, 0}=\sigma_{\text {tot }}(\omega) \mathbf{E}_{0}$, where $\mathbf{J}_{\text {tot }}(t)=\operatorname{Re}\left(\mathbf{J}_{\text {tot }, 0} e^{i \omega t}\right)$ and similarly for the electric field.

The complex frequency-dependent relative dielectric constant $\epsilon(\omega)$ is defined by (where $\epsilon_{0}$ is the vacuum permittivity)

\footnotetext{
1" It is vain to do with more what can be done with fewer" (Russell, 1946).
} 


$$
\sigma_{\text {tot }}(\omega)-\sigma_{\text {tot }}(0)=i \omega[\epsilon(\omega)-1] \epsilon_{0} .
$$

If there are no free charges $\sigma_{\text {tot }}(0)=0$ and $\mathbf{J}_{\text {tot }}=\dot{\mathbf{P}}$, where $\mathbf{P}$ is the dipole density. In this case Eq. (1) reduces to the standard definition of the frequency-dependent dielectric constant: $\mathbf{D}_{0}=\epsilon(\omega) \epsilon_{0} \mathbf{E}_{0}$, where $\mathbf{D}_{0}$ is the complex amplitude of the displacement vector $\mathbf{D}=\epsilon_{0} \mathbf{E}+\mathbf{P}$.

The negative imaginary part of $\epsilon(\omega)$ is referred to as the "dielectric loss" because it determines the dissipation in excess of the dc dissipation. ${ }^{2}$ The dielectric loss in disordered solids usually exhibits a peak, much like that characterizing dielectric relaxation in dipolar liquids (Böttcher and Bordewijk, 1978).

At the frequencies of interest here (much below phonon frequencies, $\omega \ll 10^{13} \mathrm{~Hz}$ ) it is generally believed that the bound charge polarization may be regarded as instantaneous. ${ }^{3}$ This implies that the bound charge dielectric constant $\epsilon_{\infty}$ is frequency independent. The bound charge contribution to $\sigma_{\text {tot }}(\omega)$ is $i \omega\left(\epsilon_{\infty}-1\right) \epsilon_{0}$. The ac conductivity $\sigma(\omega)$ is by definition the free charge carrier contribution to $\sigma_{\text {tot }}(\omega)$. Since $\sigma_{\text {tot }}(\omega)=\sigma(\omega)$ $+i \omega\left(\epsilon_{\infty}-1\right) \epsilon_{0}$ Eq. (1) implies

$$
\sigma(\omega)-\sigma(0)=i \omega\left[\epsilon(\omega)-\epsilon_{\infty}\right] \epsilon_{0} .
$$

The real part of $\sigma(\omega)$ is usually denoted by $\sigma^{\prime}(\omega)$ and the imaginary part by $\sigma^{\prime \prime}(\omega)$. Clearly, $\sigma^{\prime \prime}(\omega) \neq 0$ reflects a phase difference between field and free charge current. Below phonon frequencies, whenever the conductivity is frequency dependent the charge carrier displacement always lags behind the electric field. This time lag is at most one-quarter of a period, so the current reaches its maximum earlier than the field, implying $\sigma^{\prime \prime}(\omega)>0$. In the language of electronics this reflects a capacitive response rather than an inductive one. Of course, $\sigma^{\prime}(\omega)$ is also positive because thermodynamics requires positive dissipation.

\section{AC CONDUCTION IN DISORDERED SOLIDS: FACTS}

Solids are classified into metal and nonmetals. A metal has a large weakly temperature-dependent dc conductivity, a nonmetal has a much smaller dc conductivity which, however, increases strongly with increasing temperature (Kittel, 1996). Only for disordered nonmetals is ac conduction different from dc observed far below phonon frequencies. As mentioned in the Introduction, these solids have quite similar ac conductivities, "universality." Examples of solids with universal ac properties are ion conducting glasses (Owen, 1963; Tomozawa, 1977; Angell, 1990; Kahnt, 1991; Roling, 1998), amorphous semiconductors (Mott and Davis, 1979; Long, 1982; Elliott, 1990; Long, 1991), polycrystalline semiconductors (Kuanr and Srivastava, 1994), electron conduct-

\footnotetext{
${ }^{2}$ Recall that the average power absorbed per unit volume is the real part of $\sigma_{\text {tot }}(\omega)\left|\mathbf{E}_{0}\right|^{2} / 2$.

${ }^{3}$ This is true in disordered solids only when one ignores secondary relaxations like those due to two-level tunneling systems important at very low temperatures.
}

ing polymers (Epstein, 1986; Jastrzebska, Jussila, and Isotalo, 1998), ion conducting polymers (Rozanski et al., 1995), transition metal oxides (Namikawa, 1975; Mansingh, 1980; Suzuki, 1980), metal cluster compounds (van Staveren, Brom, and de Jongh, 1991), organicinorganic composites (Bianchi et al., 1999), and doped single-crystal semiconductors at helium temperatures (where the disorder due to the random positions of the doping atoms becomes important) (Pollak and Geballe, 1961).

Figure 1 shows ac data for six different disordered solids, three ion conducting (a)-(c) and three electron conducting (d)-(f) [Fig. 1(b) is actually for a highly viscous ionic melt just above the glass transition]. Clearly, the ac conductivities are quite similar. There are numerous other examples. While ion conduction is a classical barrier crossing process, electron conduction in disordered solids usually proceeds via quantum-mechanical tunneling between localized states (i.e., states with wave functions decaying exponentially in space). What do these conduction mechanisms have in common in disordered solids? The most likely answer is: very broad distributions of jump rates/tunneling rates/local mobilities (Dyre, 1988; Elliott, 1990). Below we study two models with such broad distributions and find that they roughly reproduce experiment.

Ac universality was first discovered for ion conducting oxide glasses. Taylor $(1956,1957,1959)$ showed that the dielectric loss for different glasses fell on a single plot against scaled frequency. He also noted that the activation energy of the dc conductivity was the same as that of the frequency marking the onset of ac conduction. Subsequently, Isard (1961) relabeled Taylor's axis by plotting dielectric loss against the log of the product of frequency and dc resistivity, equivalent to the following representation:

$$
\widetilde{\sigma} \equiv \sigma(\omega) / \sigma(0)=F\left(C \frac{\omega}{\sigma(0)}\right) .
$$

Since then Eq. (3), which we shall refer to as "TaylorIsard scaling," has been used by several authors. For instance, Taylor-Isard scaling was used by Scher and Lax (1973) in their famous papers on the continuous time random-walk approximation, by Balkan et al. (1985) for amorphous semiconductors, by Summerfield (1985) for hopping models solved in a certain approximation, and by van Staveren, Brom, and de Jongh (1991) for metal cluster compounds. Brom et al. (1998) discuss the generality of Taylor-Isard scaling for electron conducting disordered solids. For ion conducting glasses Taylor-Isard scaling was used recently by Kulkarni, Lunkenheimer, and Loidl (1998), and by Roling (1998).

Three examples of Taylor-Isard scaling are shown in Fig. 2. Figures 2(a) and (b) give the master curves for the data shown in Figs. 1(a) and (d). Figure 2(c) shows the master curves for eight different ion conducting glasses. The master curves in Fig. 2 are similar, but not identical. In all three examples the authors chose to fix the Taylor-Isard scaling constant $C$ to be proportional to $1 / T$. As shown below [Eq. (7)], in general $C \propto \Delta \epsilon$ where 


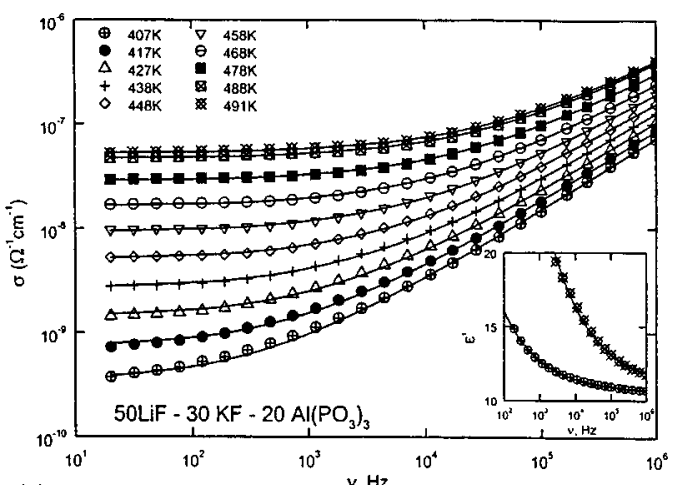

(a)
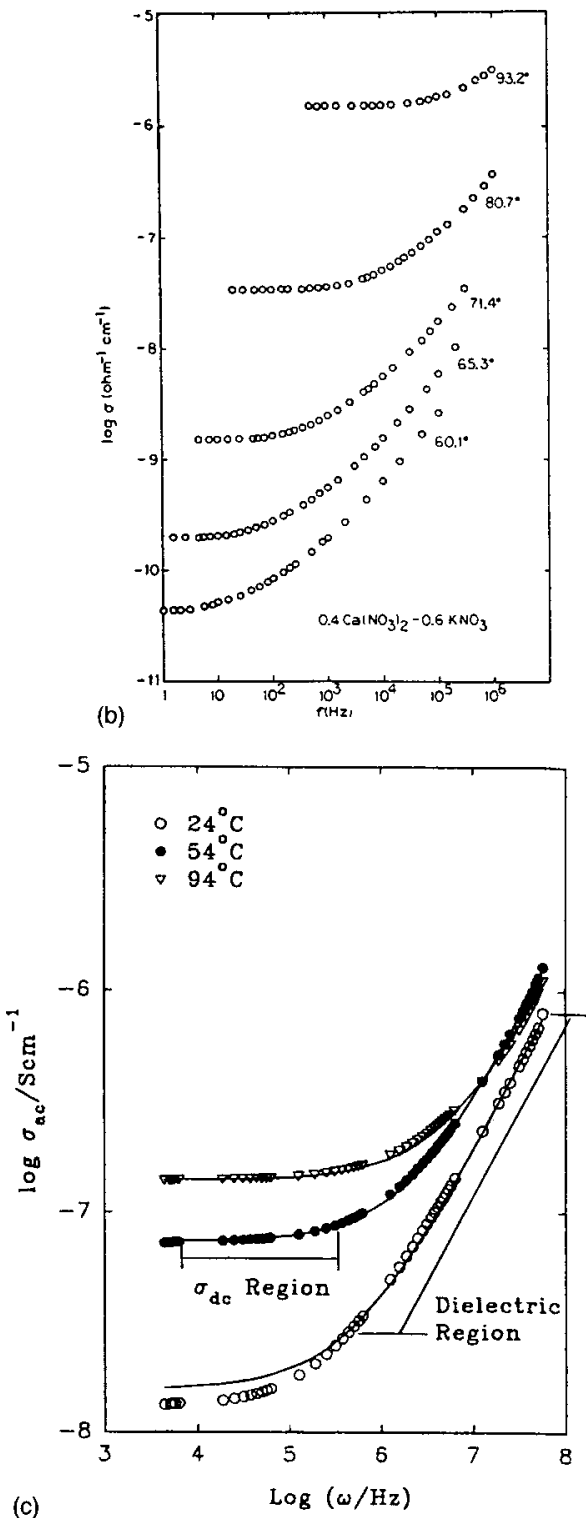
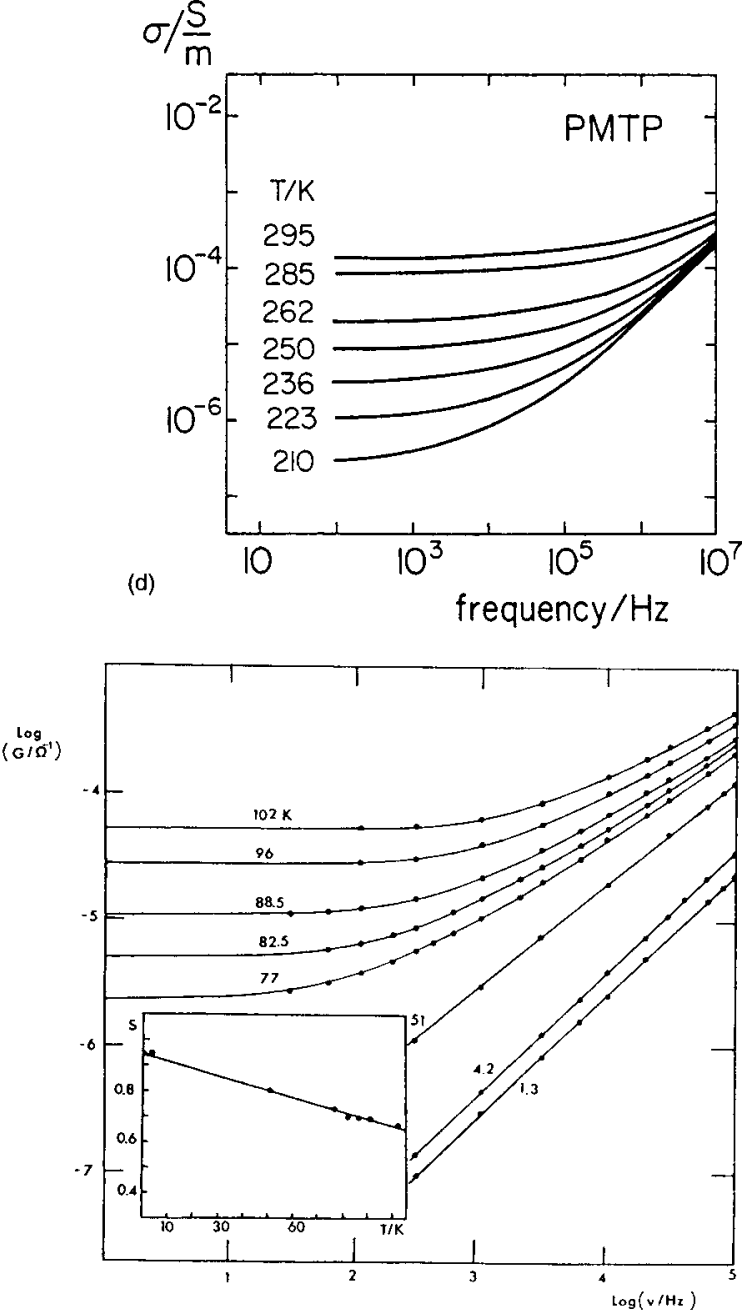

(e)

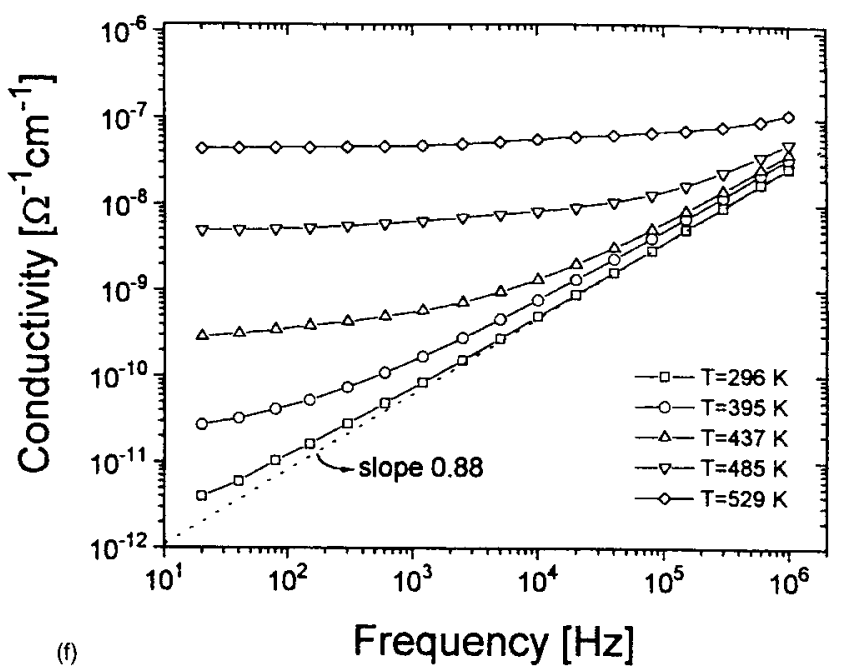

FIG. 1. Ac conductivity of typical ion (a)-(c) and electron (d)-(f) conducting disordered solids. Each figure shows a log-log plot of the real part of the conductivity as a function of frequency at various temperatures. At low frequencies the conductivity is constant, at high frequencies it follows an approximate power law with exponent below one. Data are shown for the following systems: (a) 50LiF-30KF-20Al $\left(\mathrm{PO}_{3}\right)_{3}$ glass (inset: real part of the dielectric constant as a function of frequency) (Kulkarni, Lunkenheimer, and Loidl, 1998). (b) $0.4 \mathrm{Ca}\left(\mathrm{NO}_{3}\right)_{2}-0.6 \mathrm{KNO}_{3}$ highly viscous melt (Howell et al., 1974). (c) Thermoplastic polyurethane doped with $\mathrm{NH}_{4} \mathrm{CF}_{3} \mathrm{SO}_{3}$ (van Heumen et al., 1995). (d) Poly(methylthiophene) (Rehwald, Kiess, and Binggeli, 1987). (e) Amorphous germanium film (inset: approximate power-law exponent - here labeled $s$-as a function of temperature) (Long and Balkan, 1980). (f) Polycrystalline diamond film (Fiegl et al., 1994). 

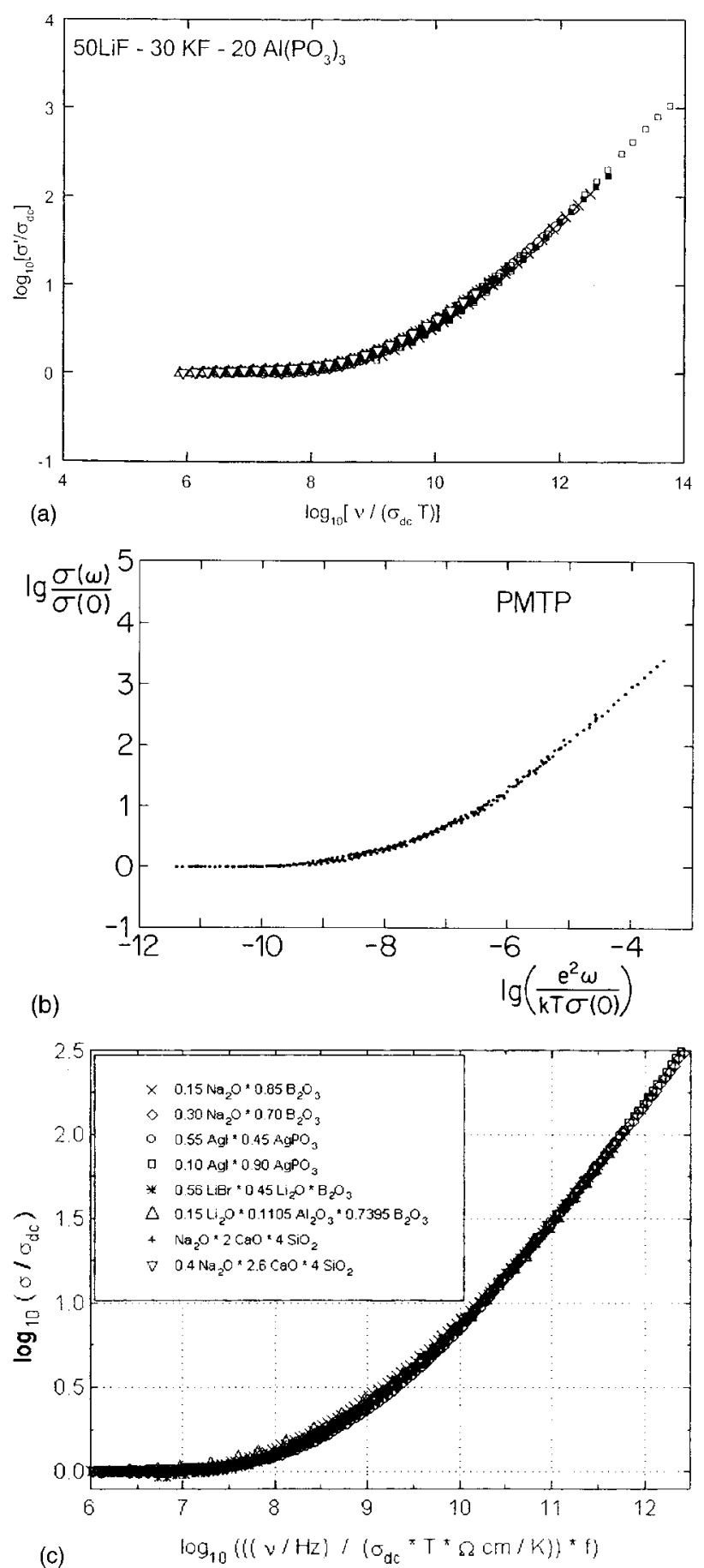

FIG. 2. Ac conductivity master curves. Each figure shows a $\log -\log$ plot of the real part of the normalized ac conductivity [in the text denoted by $\widetilde{\sigma}^{\prime}$ ] as a function of scaled frequency. (a) gives the master curve for the ion conducting glass data of Fig. 1(a) (Kulkarni, Lunkenheimer, and Loidl, 1998); (b) gives the master curve for the electron conducting polymer data of Fig. 1(d) (Rehwald, Kiess, and Binggeli, 1987); (c) gives master curves for data on eight different ion conducting oxide glasses (Roling, 1998).

$\Delta \epsilon=\epsilon(0)-\epsilon_{\infty}$; thus choosing $C \propto 1 / T$ corresponds to assuming a "Curie law" for $\Delta \epsilon$. This law, however, is not always obeyed (Namikawa, 1975) so possibly even better master curves would have been achieved if no Curie law was assumed.
We now list the ac characteristics of the vast majority of disordered solids. When reading the points below it may be helpful to compare to Figs. 1 and 2 .

(1) The real part of the ac conductivity increases with frequency, the imaginary part is non-negative.

(2) At high frequencies $\sigma^{\prime}(\omega)$ follows an apparent power law,

$$
\sigma^{\prime}(\omega) \propto \omega^{n}
$$

(3) Deviations from this power law correspond to $n$ increasing weakly with frequency.

(4) $n$ is between 0.6 and 1.0 .

(5) In a fixed frequency range $n$ increases as temperature decreases and $n \rightarrow 1.0$ for $T \rightarrow 0$.

(6) When there is no measurable dc conductivity $n$ is close to 1.0 .

(7) At low frequencies there is a gradual transition to frequency-independent conductivity.

(8) In a log-log plot the power-law regime of the ac conductivity is much less temperature dependent than the dc conductivity.

(9) When $n$ is close to $1.0 \sigma^{\prime}(\omega)$ is almost temperature independent.

(10) The ac conductivity obeys time-temperature superposition, i.e., the shape of $\sigma^{\prime}(\omega)$ in a log-log plot is temperature independent. This makes it possible to construct a master curve. Time-temperature superposition applies also to $\sigma^{\prime \prime}(\omega)$.

(11) The shape of the master curve is roughly the same for all disordered solids (universality).

(12) Whenever $\sigma(0)$ is measurable there is a dielectric loss peak.

(13) The onset of ac conduction takes place around the dielectric loss peak frequency $\omega_{m}$.

(14) $\omega_{m}$ satisfies the Barton-Nakajima-Namikawa relation (Barton, 1966; Nakajima, 1972; Namikawa, 1975)

$$
\sigma(0)=p \Delta \epsilon \epsilon_{0} \omega_{m}
$$

where $p$ is a numerical constant of order 1. Experimental evidence for Eq. (5) is reproduced in Fig. 3.

(15) The dielectric loss strength $\Delta \epsilon$ is much less temperature dependent than $\omega_{m}$ or $\sigma(0)$, so Eq. (5) implies the rough proportionality

$$
\sigma(0) \sim \omega_{m}
$$

(16) $\sigma(0)$ and $\omega_{m}$ are Arrhenius temperature dependent with the same activation energy.

Is not time-temperature superposition inconsistent with the finding that $n$ depends on temperature? The answer is no because the power-law description is only approximate. Thus if temperature is lowered the master curve is displaced to lower frequencies (compare Fig. 1); at the same time $n \rightarrow 1.0$ for measurements performed in a fixed frequency range. We conclude that the slope of the master-curve in a log-log plot-instead 


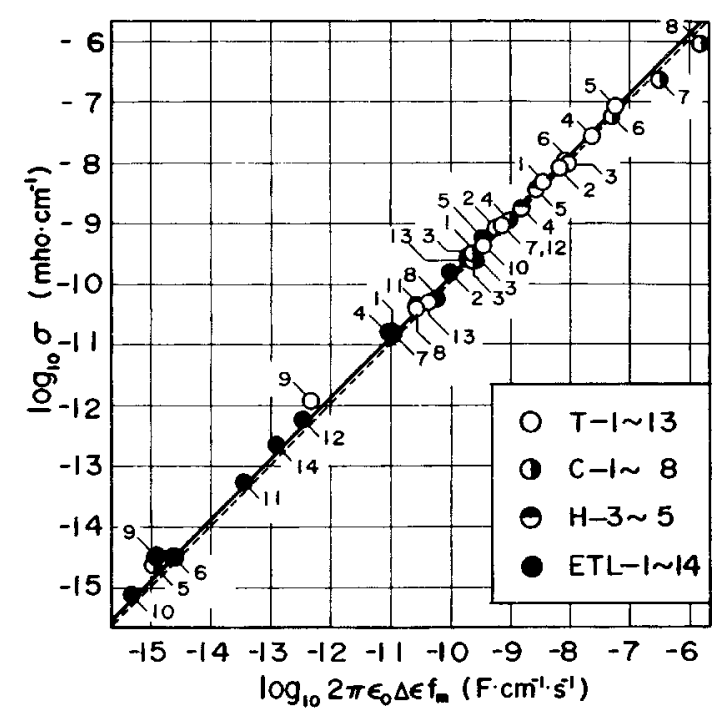

FIG. 3. Test of the Barton-Nakajima-Namikawa relation [Eq. (5), note that $2 \pi f_{m}=\omega_{m}$ ] for 40 different alkali ion conducting oxide glasses. The dashed line corresponds to $p=1$ in Eq. (5), the solid line gives the best fit to data. These data from four different laboratories were compiled by Nakajima (1972) who presented a similar figure for 14 electron conducting transitionmetal oxide glasses.

of being constant as for an exact power-law-goes to one as scaled frequency goes to infinity. ${ }^{4}$

Time-temperature superposition makes it possible to determine the Taylor-Isard scaling constant $C$ of Eq. (3) and derive the Barton-Nakajima-Namikawa relation. We first find $C$ by proving the scaling recently proposed by Sidebottom (1999):

$$
\widetilde{\sigma}=F\left(\Delta \epsilon \epsilon_{0} \frac{\omega}{\sigma(0)}\right) \text {. }
$$

If $\widetilde{\omega}$ is the scaled frequency, the master curve $\widetilde{\sigma}(\widetilde{\omega})$ is expanded to first order: $\widetilde{\sigma}=1+i \widetilde{\omega} A$. Since $\sigma=\widetilde{\sigma} \sigma(0)$ we have $\sigma(\omega)=\sigma(0)+i \widetilde{\omega} A \sigma(0)$. On the other hand, Eq. (2) implies to first order in frequency $\sigma(\omega)=\sigma(0)$ $+i \omega \Delta \epsilon \epsilon_{0}$. Comparing these two expressions leads to $\widetilde{\omega}=A^{-1}\left[\Delta \epsilon \epsilon_{0} / \sigma(0)\right] \omega$, and consequently $C=\Delta \epsilon \epsilon_{0}$ in Eq. (3) [the numerical value of $A$ is of no significance in this proof] (Schrøder and Dyre, 2000).

Once Eq. (7) has been derived, the Barton-NakajimaNamikawa relation Eq. (5) follows: Equation (2) implies $\epsilon(\omega)-\epsilon_{\infty} \propto(\widetilde{\sigma}-1) / \widetilde{\omega}$ which is a function of $\widetilde{\omega}$. Thus the dielectric loss has its maximum at some particular temperature-independent value of $\widetilde{\omega}$. Denoting this by $\widetilde{\omega}_{m}$, we have from the above derivation of Eq. (7) $A \widetilde{\omega}_{m}=\left[\Delta \epsilon \epsilon_{0} / \sigma(0)\right] \omega_{m}$. This is precisely the required Eq. (5) if $p$ is defined by $1 / p=A \widetilde{\omega}_{m}$. We cannot prove from general arguments, of course, the experimental finding that $p$ is never far from one (Namikawa, 1975).

\footnotetext{
${ }^{4}$ This all applies far below phonon frequencies. Around phonon frequencies there are various resonance phenomena and at even higher frequencies $\sigma^{\prime}(\omega)$ goes to zero fast enough that $\int_{0}^{\infty} \sigma^{\prime}(\omega) d \omega$ is finite (Kubo, 1957).
}

We finally note that there are exceptions to the above 16 points. There are solids where $n$ is slightly larger than 1.0 (Lakatos and Abkowitz, 1971; Durand et al., 1994; Cramer and Buscher, 1998). There are solids where the dc conductivity is not Arrhenius, for instance group-IV amorphous semiconductors (Mott and Davis, 1979) or fast ion conducting glasses (Angell, 1990). Finally, in some cases the conductivity is weakly frequency dependent much below $\omega_{m}$ (Jonscher, 1996). In our opinion these are insignificant exceptions to the overall experimental picture, but this viewpoint is not universally accepted. Thus Elliott (1994), Macdonald (1997), and Ngai and Moynihan (1998) all emphasize the differences between various disordered solids. On the other hand, an experienced experimentalist in this field recently discontinued routine ac measurements because "we always see more or less the same" (Kremer, 1999). Why is that? Is there a simple way of understanding ac universality? These questions motivate the study of the two simple models in the following.

\section{MACROSCOPIC MODEL}

The first model we consider assumes the disorder is present only on a macroscopic scale.

\section{A. Definition}

Any solid consisting of phases with different conductivity has an overall conductivity which increases with frequency (Maxwell, 1891; Wagner, 1913). This is because at high frequencies localized charge carrier motion makes it possible to take maximum advantage of well conducting regions, while at lower frequencies charge transport must extend over longer distances and is limited by bottlenecks of poorly conducting regions.

Consider a solid with spatially varying, frequencyindependent free charge conductivity $g(\mathbf{r})$, but uniform bound charge dielectric constant $\epsilon_{\infty}$. If $\mathbf{J}$ is the free charge current density and $\mathbf{D}$ the displacement vector, we have $\mathbf{J}(\mathbf{r}, t)=g(\mathbf{r}) \mathbf{E}(\mathbf{r}, t)$ and $\mathbf{D}(\mathbf{r}, t)=\epsilon_{\infty} \epsilon_{0} \mathbf{E}(\mathbf{r}, t)$. Combining these equations with (i) the definition of the electrostatic potential $\phi[\mathbf{E}=-\nabla \phi]$, (ii) Gauss' law $[\nabla \cdot \mathbf{D}$ $=\rho$, where $\rho$ is the free charge density], and (iii) the continuity equation $[\dot{\rho}+\nabla \cdot \mathbf{J}=0]$, leads to the following equation for $\phi$ in a periodic field (Fishchuk, 1986; Dyre, 1993)

$$
\nabla \cdot\left\{\left[i \omega \epsilon_{\infty} \epsilon_{0}+g(\mathbf{r})\right] \nabla \phi\right\}=0 .
$$

It is not difficult to show that when Eq. (8) is discretized one arrives at the circuit shown in Fig. 4 (Fishchuk, 1986; Dyre, 1993). All capacitors in the circuit are equal, proportional to $\epsilon_{\infty} \epsilon_{0}$, while the resistors are proportional to the local resistivity $1 / g(\mathbf{r})$.

It is tempting to interpret the resistor currents as the free charge currents and the capacitor currents as the bound charge currents. This is not correct, however, because in that case according to Kirchhoff's current law there could be no charge accumulation at any node of the circuit, i.e., anywhere in the solid. So how is the 


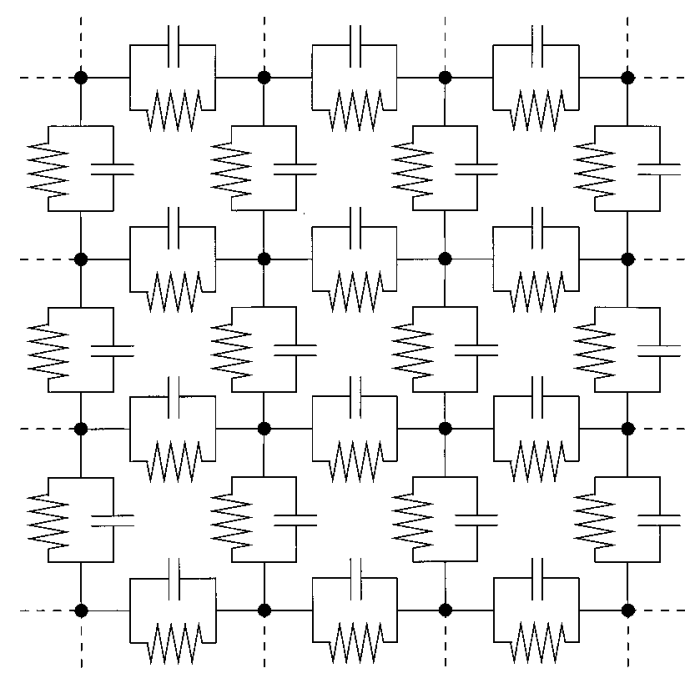

FIG. 4. Electrical equivalent circuit arising from the discretization of Eq. (8), the basic equation for an inhomogeneous solid in an ac electric field. This circuit and its higher dimensional analogs describe the macroscopic model (Fishchuk, 1986; Dyre, 1993). All capacitors are equal, proportional to the bound charge dielectric constant, while each resistor is proportional to the inverse free charge conductivity at the corresponding position in the solid. When the circuit is subjected to a potential difference applied to two opposing faces the electrostatic potentials at the nodes are found by solving Kirchhoff's equations. As shown in the text, the resistor currents are the free charge currents and the capacitor currents are Maxwell's displacement currents. The bound charge currents are only parts of this, the remainder is not associated with charge transport (Reitz, Milford, and Christy, 1993).

circuit to be interpreted? Surely, the resistor currents are the free charge currents-this follows simply from the definition of the resistors as being proportional to the local free charge resistivity. But the capacitor currents are less straightforward: The charge on any capacitor is the capacity $\left(\propto \epsilon_{\infty} \epsilon_{0}\right)$ times the potential drop across the capacitor. In a periodic field the current through the capacitor is therefore proportional to $i \omega \epsilon_{\infty} \epsilon_{0}$ times the potential drop. Since the displacement vector is given by $\mathbf{D}=\epsilon_{\infty} \epsilon_{0} \mathbf{E}$ and the electric field is proportional to the potential drop, we see that the capacitor current is proportional to $\dot{\mathbf{D}}$. This time derivative is by definition Maxwell's displacement current (Reitz, Milford, and Christy, 1993). When the proportionality constants are properly included in the calculation one finds that the capacitor current is precisely the displacement current (Dyre, 1993). Note that the capacitors would be there even in the absence of bound charges, i.e., when $\epsilon_{\infty}=1$.

To extract the overall ac conductivity from the circuit one imagines a periodic potential applied across two opposing faces acting as electrodes. The average resistor current determines the free charge ac conductivity $\sigma(\omega)$. The admittance (i.e., the reciprocal of the impedance) between the electrodes is denoted by $Y(\omega)$. If $L$ is the linear dimension of the circuit and $d$ the spatial dimension, $\sigma(\omega)$ is found by subtracting the capacitor contribution to $Y(\omega)$ (Fishchuk, 1986; Dyre, 1993):

$$
\sigma(\omega)=\frac{Y(\omega)}{L^{d-2}}-i \omega \epsilon_{\infty} \epsilon_{0} .
$$

The frequency dependence of $\sigma$ is not due to the (subtracted) direct capacitor contribution to the overall admittance. Instead, $\sigma(\omega) \neq \sigma(0)$ because the capacitors influence the node potentials which determine the resistor currents.

To realistically model a disordered nonmetal, the local free charge conductivity is taken to be Arrhenius temperature dependent: $g=g_{0} \exp (-\beta E)$ where $\beta$ $=1 / k_{B} T$ and $E$ is the so-called activation energy. Conduction may be classical or quantum mechanical, it makes no difference in the present treatment. The solid disorder is reflected in $g=g(\mathbf{r})$ being somehow random. ${ }^{5}$ A random function typically has a correlation length beyond which values are essentially uncorrelated. If the discretization length is chosen as this correlation length, it makes good sense to assume the resistors are uncorrelated from link to link. This assumption allows the model to be uniquely defined by the activation energy probability distribution $p(E)$. By making this assumption we ignore the details of the "microgeometry" (Baskin et al., 1997).

In one dimension the circuit is a series of $R C$ elements and the calculation of $Y(\omega)$ is straightforward. In particular, the circuit impedance at zero frequency is the sum of the individual resistors, implying $\sigma(0)$ $=\left\langle g^{-1}\right\rangle^{-1}$ (Dyre, 1993). In more than one dimension the calculation of $\sigma(\omega)$ involves solving Kirchhoff's circuit equations. This cannot be done analytically, even in the dc limit. In the high-frequency limit, however, the capacitor admittances are so large that they completely dominate, resulting in a spatially homogeneous electric field. Consequently, the average resistor current is determined by the average free charge conductivity, i.e., $\sigma(\infty)=\langle g\rangle$ (Dyre, 1993). Summarizing the exact results,

$$
\begin{aligned}
& d=1: \quad \sigma(0)=\left\langle g^{-1}\right)^{-1}, \\
& d \geqslant 1: \quad \sigma(\infty)=\langle g\rangle .
\end{aligned}
$$

\section{B. Ac universality in the extreme disorder limit}

If temperature is lowered, $\beta$ increases and the local conductivities cover more and more decades: The extreme disorder limit is approached. ${ }^{6}$ Although not obvi-

\footnotetext{
${ }^{5} \mathrm{~A}$ disordered solid is rarely random in the mathematical sense (Ziman, 1979). We here follow what has become a standard approach, namely to replace complexity by randomness. The rationale for doing so has been beautifully summarized by Wolynes (1992).

${ }^{6}$ If $W$ is a dimensionless measure of the degree of disorder, $W \ll 1$ corresponds to weak disorder while $W \gg 1$ corresponds to strong disorder. The term "extreme disorder" is used here for the case $\ln (W) \gg 1$. We prefer to speak of the extreme disorder limit, but mathematically, of course, this is the same as the strong disorder limit $(W \rightarrow \infty)$.
} 
ous a priori, it turns out that in this limit the ac conductivity in scaled units becomes independent of both $\beta$ and $p(E)$. This is ac universality as the term is used here for models. No rigorous mathematical proof of ac universality exists, but there is convincing evidence from three sources: (i) ac universality is predicted by analytical approximations; (ii) ac universality is found in computer simulations; and (iii) it is possible to physically understand the origin of ac universality. Points (i) and (ii) are considered below for the macroscopic model and in Sec. $\mathrm{V}$ for the symmetric hopping model. Point (iii) is discussed in Sec. VI for both models.

To find $\sigma(\omega)$ we need to calculate the overall admittance of the circuit in Fig. 4. For this we shall use the effective-medium approximation (EMA), a standard technique for calculating average physical properties of random mixtures. The idea is to focus on one small part of the mixture and regard it as embedded in an effective medium with the average property. Then selfconsistency is required such that, on the average, the embedding in the effective medium has the same overall property as the effective medium itself (Landauer, 1978; Bergman and Stroud, 1992). This approximation was first used by Bruggeman (1935) for calculating the dielectric constant of mixtures of dielectrics, and for calculating the thermal and dc electrical conductivity of mixtures. The effective-medium approximation may also be used for calculating the bulk and shear moduli of a mixture of solids with different elastic properties (Berryman, 1980) or, as in the next section, the ac conductivity of a hopping model. The idea has even been used for calculating the quantum-mechanical average oneparticle Green's function for a disordered system, in this situation referred to as the "coherent potential approximation" (Economou, 1983).

According to the effective-medium approximation for randomly varying admittances $y$ on a cubic lattice in $d$ dimensions, the overall admittance is the same as that of a circuit of identical admittances $y_{m}$, where $y_{m}$ is the solution (Kirkpatrick, 1973) of

$$
\left\langle\frac{y-y_{m}}{y+(d-1) y_{m}}\right\rangle=0 .
$$

The brackets indicate averaging over the admittance probability distribution $p(y)$. To apply Eq. (11) to the macroscopic model, one substitutes the admittance of each $R C$ element of Fig. 4, $y \propto g(\mathbf{r})+i \omega \epsilon_{\infty} \epsilon_{0}$, into Eq. (11). Note that $y_{m}$ becomes frequency dependent. Equation (9) implies $y_{m} \propto \sigma(\omega)+i \omega \epsilon_{\infty} \epsilon_{0}$. Thus the following equation for $\sigma=\sigma(\omega)$ is arrived at (Fishchuk, 1986; Dyre, 1993):

$$
\left\langle\frac{g-\sigma}{g+(d-1) \sigma+d i \omega \epsilon_{\infty} \epsilon_{0}}\right\rangle=0 .
$$

The brackets indicate averaging over $p(g)$.

A straightforward calculation shows that in one dimension the dc conductivity is correctly predicted by Eq.
(12). Equation (12) also correctly implies $\sigma(\infty)=\langle g\rangle$ in any dimension: At very large frequencies the relative variation of the denominator is small. Consequently, the denominator may be regarded as a constant $C$, and Eq. (12) reduces to $\langle[g-\sigma(\infty)] / C\rangle=0$ which implies $\sigma(\infty)$ $=\langle g\rangle$.

It is possible to simplify Eq. (12) in the extreme disorder limit (Dyre, 1993). In terms of a suitably defined dimensionless frequency $\widetilde{\omega}$, for any continuous finite width $p(E)$ the normalized ac conductivity $\widetilde{\sigma}$ is the solution of

$$
\widetilde{\sigma} \ln \widetilde{\sigma}=i \widetilde{\omega}
$$

This equation was originally derived for the hopping model describing a dilute system of electrons tunneling between states randomly localized in space (Bryksin, 1980; Böttger and Bryksin, 1985; Long, 1991). Subsequently, Fishchuk (1986) derived Eq. (13) for the macroscopic model with "box" activation energy distribution $[p(E)$ flat with sharp cutoffs].

The effective-medium approximation universality equation, Eq (13), is easy to solve numerically. An accurate analytical approximation to the solution is available (Dyre, 1993); it is even possible to give an explicit integral representation of $\widetilde{\sigma}(\widetilde{\omega})$ (Dyre and Jacobsen, 1995). The solution is constant at low frequencies $(\widetilde{\omega} \ll 1)$. At high frequencies $(\widetilde{\omega} \gg 1)$ the real part of the conductivity follows an approximate power law with exponent $n<1$ which slowly goes to one as $\widetilde{\omega} \rightarrow \infty$ (Bryksin, 1980). Note that, while the ac conductivity at any finite temperature is constant at sufficiently high frequencies [Eq. (10)], the limit function $\widetilde{\sigma}(\widetilde{\omega})$ diverges as $\widetilde{\omega} \rightarrow \infty$.

How well do the effective-medium predictions hold? Figure 5 reproduces examples of simulations in two dimensions. Only imaginary frequencies (denoted by $s$ ) were used in these simulations. ${ }^{7}$ This is a technical trick which simplifies calculations_-at imaginary frequencies all numbers are real because the capacitor admittances are real. Figure 5(a) shows results for one activation energy distribution at different $\beta$ 's, clearly converging as $\beta \rightarrow \infty$. The solid curves show the predictions of the effective-medium approximation at finite temperatures [Eq. (12) solved numerically]. Similar results are found for other distributions (Dyre, 1993). Figure 5(b) shows the ac conductivity for different distributions at $\beta=160$ (in dimensionless units). All distributions lead to the same ac conductivity, which is well represented by Eq. (13) (solid curve). A few simulations in three dimensions were presented by Dyre (1993) but extensive simulations are still lacking. The best results presently available seem to be those of Riedel and Dyre (1994) who,

\footnotetext{
${ }^{7}$ The ac conductivity is an analytic function of frequency. Consequently, a knowledge of this function on just one line in the complex plane by "analytic continuation" determines the function uniquely (Churchill and Brown, 1984). In particular, knowing the ac conductivity at imaginary frequencies determines it at real, physical frequencies.
} 


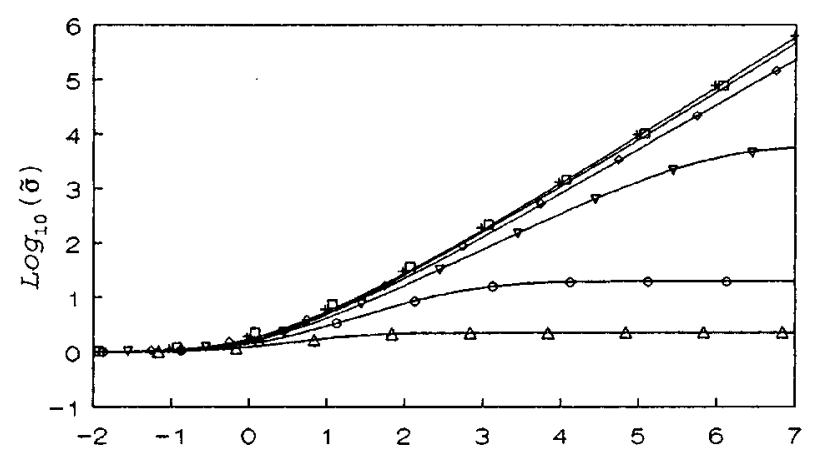

(a)

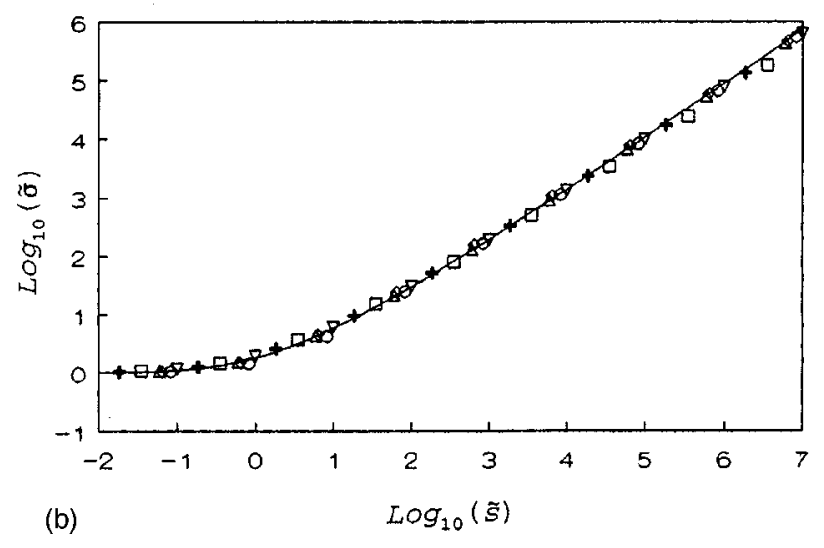

FIG. 5. Computer simulations of the macroscopic model's ac conductivity at imaginary frequencies in two dimensions [reproduced from Dyre (1993)]. (a) shows a log-log plot of $\widetilde{\sigma}$ as a function of scaled imaginary frequency $\widetilde{s}$ [scaling defined in Dyre (1993)] for the activation energy probability distribution $p(E)=2 E(0 \leqslant E \leqslant 1)$ at $\beta$ equal to $5,10,20,40,80,160$ in order of increasing conductivity. The solid curves are the predictions of the effective-medium approximation at finite temperatures obtained by solving Eq. (12) numerically. (b) shows results for $\widetilde{\sigma}(\widetilde{s})$ at $\beta=160$ for the following six activation energy probability distributions: Asymmetric Gaussian $(\triangle)$, box $(+)$, Cauchy $(\bigcirc)$, symmetric exponential $(\diamond)$, power law with exponent $-4(\square)$, and triangle $(\nabla)$. At extreme disorder all distributions have the same $\widetilde{\sigma}(\widetilde{s})$. The universal ac conductivity is well represented by the effective-medium approximation universality equation, Eq. (13), which is given by the solid curve.

using a recursive technique, found good agreement with Eq. (13) in the extreme disorder limit. In conclusion, the effective-medium approximation universality equation, Eq. (13), describes the universal ac conductivity of the macroscopic model well in two and, most likely, also in three dimensions.

\section{SYMMETRIC HOPPING MODEL}

The macroscopic model does not apply for solids that are strongly disordered on the atomic scale. We now consider a model with microscopic disorder. While the macroscopic model via Gauss' law includes all Coulomb interactions, to keep things simple these interactions are ignored in the below "hopping" model.

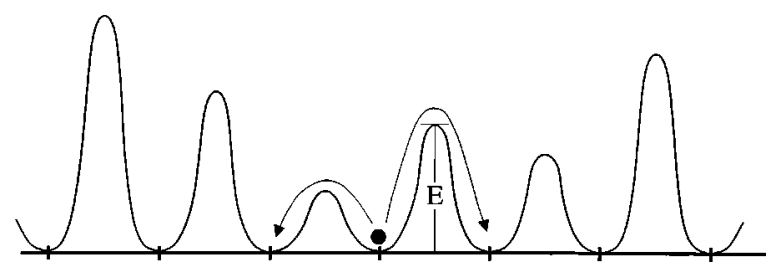

FIG. 6. Typical potential for a system described by the symmetric hopping model (random barrier model) in one dimension. The arrows indicate the two possible jumps for the charge carrier shown. The term "symmetric" refers to the fact that the jump rate is the same for jumps forwards and backwards across a given barrier. The symmetric hopping model corresponds to the discrete version of motion in this potential, where noninteracting charge carriers reside on a lattice defined by the minima. At low temperatures most time is spent close to energy minima. Occasionally, a charge carrier by chance acquires enough energy from the surrounding heat bath to jump into a neighboring minimum. If the barrier height is $E$, the probability per unit time for a jump is the attempt frequency times $\exp (-\beta E)$, where $\beta=1 / k_{B} T$. At low temperatures the charge carrier almost always chooses the lowest barrier. This implies the "bounce-back mechanism": After one jump the next jump most likely goes back again (Kimball and Adams, 1978; Funke, 1993).

\section{A. Definition}

The term hopping refers to sudden displacement of a charge carrier from one position to another close by (Böttger and Bryksin, 1985; Long, 1991). We shall only consider the simplest hopping model. This model has noninteracting charge carriers placed on a cubic lattice with only nearest-neighbor jumps allowed. The jump rates (jump probabilities per unit time) are assumed symmetric, i.e., the same for jumps forwards or backwards between two sites. The more general asymmetric hopping model has been studied as a model, e.g., for protein dynamics or viscous liquid flow [see Stein and Newman (1995) or Dyre (1995) and their references].

Figure 6 gives a one-dimensional example of the kind of potential leading to the symmetric hopping model (also referred to as the random barrier model). Each nearest-neighbor jump rate is given (Lidiard, 1957) by $\Gamma=\gamma_{0} \exp (-\beta E)$ where $\gamma_{0}$ is the so-called attempt frequency and $\beta=1 / k_{B} T .{ }^{8}$ The barrier to be overcome, the activation energy $E$, is assumed to vary randomly.

The symmetric hopping model may seem completely unrealistic for the following three reasons: (i) It ignores that charge carriers repel each other. (ii) It allows an arbitrary number of charge carriers at each site, but-

\footnotetext{
${ }^{8}$ For simplicity only hopping over a barrier is considered, but the model also describes quantum mechanical tunneling of localized electrons. In that case $\beta$ is not inverse temperature but inverse wave-function size. In, e.g., amorphous semiconductors the electrons are localized at random positions, and the jump rates vary primarily because the jump distances vary. This situation may be described by, for the present model having jumps on a regular lattice, using the correct random position jump rate probability distribution.
} 
whether the charge carriers are ions or localized electrons-there is room for just one at each site. (iii) It does not allow site energies to vary. A more realistic model has randomly varying site energies with room for just one charge carrier at each site. Surprisingly, when this "Fermi model" is linearized with respect to an external electric field, the resulting equations are the same as those of the symmetric hopping model (Miller and Abrahams, 1960; Butcher, 1974). This traditionally serves as the justification of the symmetric hopping model. The linearization, however, involves the nontrivial assumption that the occupation number $(0$ or 1$)$ may be replaced by a continuous variable (Shklovskii and Efros, 1984). Although the connection to the "Fermi" model is not rigorous, we believe that in the extreme disorder limit it is likely that these two models have identical universal ac conductivities.

Because the charge carriers by assumption are noninteracting it is enough to consider the motion of just one of them. All relevant information is contained in the so-called master equation for the probability $P_{\mathrm{s}}$ to find the charge carrier at lattice site $\mathbf{s}, P_{\mathbf{s}}$ (van Kampen, 1981). If $\Gamma\left(\mathbf{s}, \mathbf{s}^{\prime}\right)=\Gamma\left(\mathbf{s}^{\prime}, \mathbf{s}\right)$ is the rate of jumps between $\mathbf{s}$ and $\mathbf{s}^{\prime}$-nonzero only for nearest neighbor sites-the hopping master equation (Butcher, 1974; Böttger and Bryksin, 1985; Haus and Kehr, 1987; Hughes, 1996) is

$$
\frac{d}{d t} P_{\mathbf{s}}=\sum_{\mathbf{s}^{\prime}} \Gamma\left(\mathbf{s}, \mathbf{s}^{\prime}\right)\left(P_{\mathbf{s}^{\prime}}-P_{\mathbf{s}}\right)
$$

From the values of the site probabilities at any given time Eq. (14) determines these probabilities at later times. Anyone who feels uncomfortable thinking about a time-dependent probability for one single charge carrier may instead imagine numerous noninteracting charge carriers hopping all over the lattice and define $P_{\mathbf{s}}$ as the number of charge carriers at site $\mathbf{s}$ relative to the total number. With this ensemble interpretation Eq. (14) describes the rate of change of average site occupations.

Equation (14) applies when there is no external electric field. In a nonzero field, jumps in the field direction are favored. The result is a net current. The fluctuationdissipation theorem expresses the frequency-dependent conductivity in terms of the equilibrium (i.e., zero-field) current autocorrelation function (Kubo, 1957; Becker, 1967). If $q$ is charge and $n$ is the charge carrier concentration, the fluctuation-dissipation theorem for a system of noninteracting charge carriers is

$$
\sigma(\omega)=\frac{n q^{2}}{k_{B} T} D(\omega),
$$

where the frequency-dependent diffusion constant $D(\omega)$ is defined as the Laplace transform of the velocity autocorrelation function (below $v$ is the velocity projected onto a fixed direction in space):

$$
D(\omega)=\int_{0}^{\infty}\langle v(0) v(t)\rangle e^{-i \omega t} d t .
$$

At zero frequency Eq. (16) reduces to a well-known identity for the ordinary diffusion constant and Eq. (15) reduces to the so-called Nernst-Einstein relation (Becker, 1967).
How is velocity defined for a charge carrier that virtually still sits all the time and jumps in principle infinitely fast when it finally moves? The answer is that the velocity is a sum of delta functions. This causes no problems; the velocity autocorrelation function has a $\delta(t)$ term but is otherwise continuous. A simple example is when all jump rates of Eq. (14) are equal. Jump directions are then uncorrelated and the velocity autocorrelation function is zero for $t>0$, thus proportional to $\delta(t)$. In this case $D(\omega)$ is constant and Eq. (15) implies that the conductivity is frequency independent.

Kimball and Adams (1978) derived an expression for $\sigma(\omega)$ valid for any hopping model, implying that it is always possible to write

$$
\sigma(\omega)=\sigma(\infty)-\sum_{n} \frac{A_{n}}{\gamma_{n}+i \omega},
$$

where $A_{n} \geqslant 0$ and $\gamma_{n} \geqslant 0$. Utilizing Eqs. (15) and (17), Eq. (16) by inverse Laplace transformation implies that for $t>0$

$$
\langle v(0) v(t)\rangle \leqslant 0 .
$$

In terms of the mean-square displacement $\left\langle\Delta x^{2}(t)\right\rangle$, Eq. (18) implies ${ }^{9}$ that

$$
\frac{d^{2}}{d t^{2}}\left\langle\Delta x^{2}(t)\right\rangle \leqslant 0
$$

Figure 7 illustrates the velocity autocorrelation function and the mean-square displacement. Physically, why is the velocity autocorrelation function negative for $t>0$ ? As noted Fig. 6, from any site the most likely jump is along the link with largest jump rate. The next jump is more likely to go back again than to any other site, because the link just jumped along generally has a large jump rate. This bounce-back mechanism (Kimball and Adams, 1978; Funke, 1993) explains why the velocity autocorrelation function is negative (and why the conductivity is frequency dependent).

We shall adopt the unit system in which the conductivity and the diffusion constant are both normalized such that on a homogeneous lattice with jump rate $\Gamma$ one has $\sigma=D=\Gamma$. In analogy to Eq. (10) for the macroscopic model there are two exact results for the symmetric hopping model (Alexander et al., 1981; Haus and Kehr, 1987):

$$
\begin{aligned}
& d=1: \quad \sigma(0)=\left\langle\Gamma^{-1}\right\rangle^{-1}, \\
& d \geqslant 1: \quad \sigma(\infty)=\langle\Gamma\rangle .
\end{aligned}
$$

\footnotetext{
${ }^{9}$ Squaring and averaging $\Delta x(t)=\int_{0}^{t} v\left(t^{\prime}\right) d t^{\prime}$ one arrives at $\left\langle\Delta x^{2}(t)\right\rangle=\int_{0}^{t} \int_{0}^{t}\left\langle v\left(t^{\prime}\right) v\left(t^{\prime \prime}\right)\right\rangle d t^{\prime} d t^{\prime \prime}$. The time derivative of this function has two identical contributions, each eliminating one integral, and we find $d / d t\left\langle\Delta x^{2}(t)\right\rangle=2 \int_{0}^{t}\left\langle v(t) v\left(t^{\prime \prime}\right)\right\rangle d t^{\prime \prime}$ $=2 \int_{0}^{t}\left\langle v(0) v\left(t^{\prime \prime}\right)\right\rangle d t^{\prime \prime}$ [the last equality comes from substituting $t^{\prime \prime} \equiv t-t^{\prime \prime}$ and using the identity $\left.\left\langle v(t) v\left(t-t^{\prime \prime}\right)\right\rangle=\left\langle v(0) v\left(t^{\prime \prime}\right)\right\rangle\right]$. A second differentiation leads to $d^{2} / d t^{2}\left\langle\Delta x^{2}(t)\right\rangle$ $=2\langle v(0) v(t)\rangle$.
} 

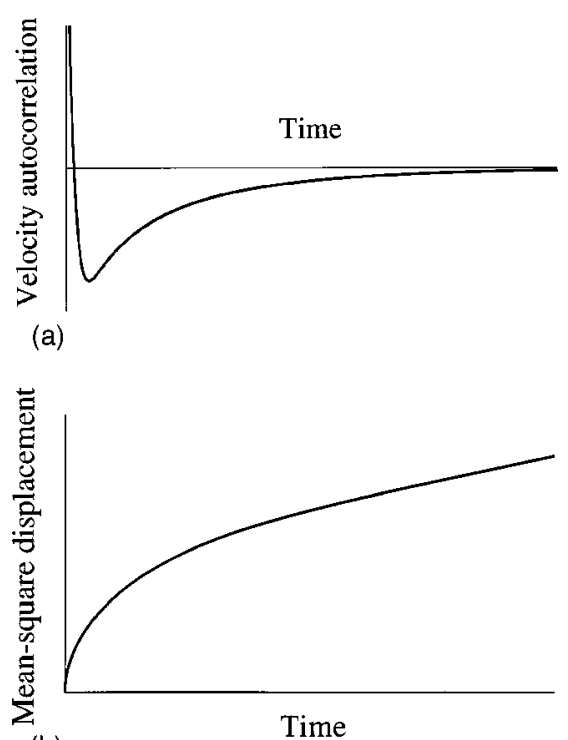

(b)

FIG. 7. Signatures of charge carrier motion in the symmetric hopping model. (a) shows the velocity autocorrelation function as a function of time. After one jump the next usually goes back again (Fig. 6). This "bounce-back mechanism" causes the velocity autocorrelation function to be negative for $t>0$ (it is always positive right at $t=0$ where it is a delta function). (b) shows the mean-square displacement as a function of time. The bounce-back mechanism is reflected in the fact that the mean-square displacement is faster at short times than it would be if the long-time behavior extrapolated linearly to zero, as it does for random walks in a homogeneous solid.

\section{B. Ac universality in the extreme disorder limit}

It is not possible to calculate the ac conductivity analytically for the symmetric hopping model, but fortunately there is an effective-medium approximation also for this model. If $\chi=\chi(\omega)$ is defined by the following $d$-dimensional integral over $\mathbf{k}=\left(k_{1}, \ldots, k_{d}\right)$ :

$$
\chi=\int_{-\pi<k_{j}<\pi} \frac{i \omega}{i \omega+2 \sigma\left[d-\Sigma_{j} \cos k_{j}\right]} \frac{d \mathbf{k}}{(2 \pi)^{d}},
$$

the effective-medium approximation self-consistency equation for $\sigma=\sigma(\omega)$ (Haus and Kehr, 1987) is

$$
\left\langle\frac{\Gamma-\sigma}{\Gamma+(d-1) \sigma+\chi(\sigma-\Gamma)}\right\rangle=0 .
$$

The brackets indicate averaging over the jump rate probability distribution $p(\Gamma)$. It is not difficult to show that Eq. (22) implies Eq. (20), just as Eq. (12) implies Eq. (10).

Equation (22) may be simplified in the extreme disorder limit $(\beta \rightarrow \infty)$. The calculations are more involved than for the macroscopic model, but the result is the same (Dyre, 1994): In terms of a suitably defined dimensionless frequency $\widetilde{\omega}, \widetilde{\sigma}(\widetilde{\omega})$ obeys the effective medium approximation universality equation, Eq. (13), independent of the activation energy probability distribution. Below two dimensions the effective-medium approximation does not lead to Eq. (13), though. This fact becomes
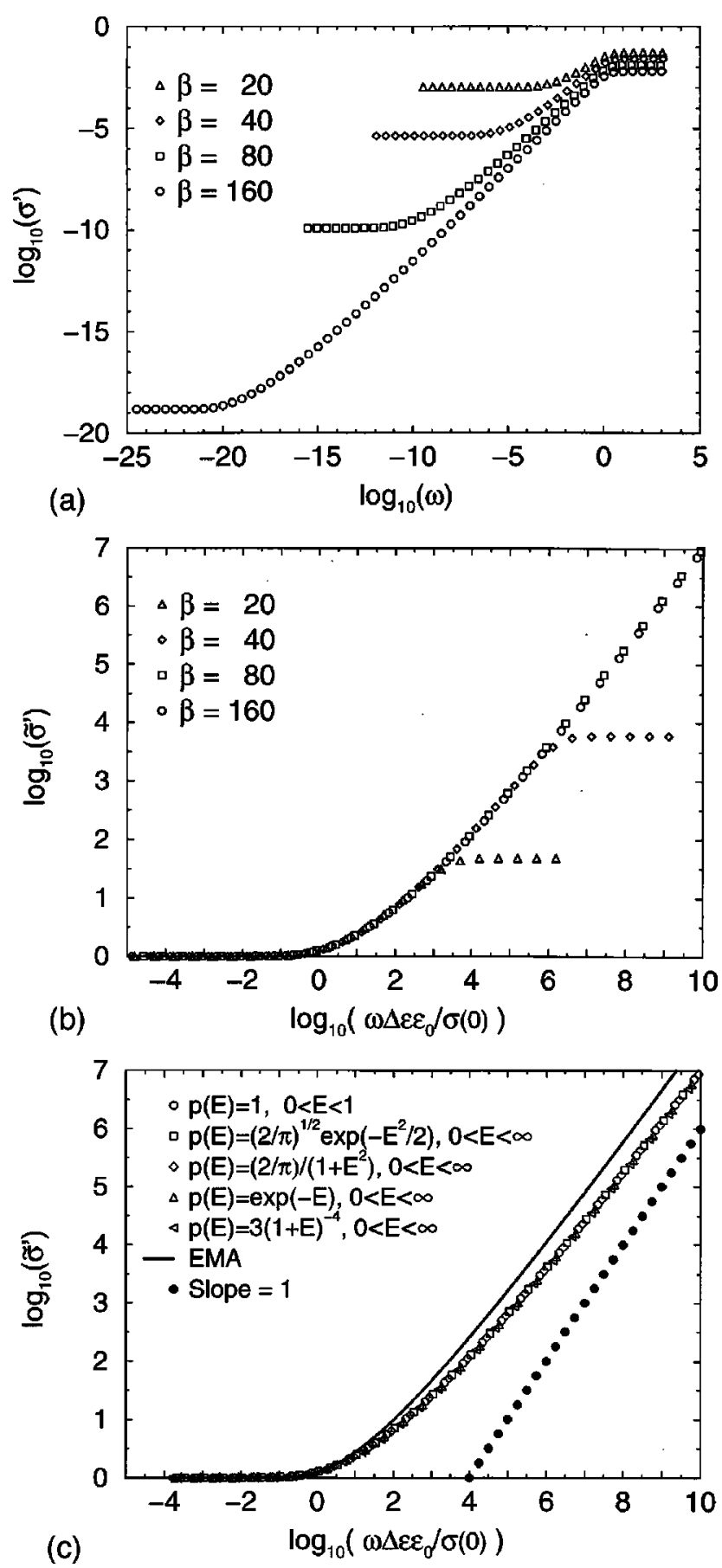

FIG. 8. Computer simulations of the symmetric hopping model in three dimensions at real frequencies (Schrøder and Dyre, 2000). The simulations were carried out by solving the hopping master equation using a new algorithm (Schrøder, 1999). (a) Real part of the ac conductivity in nonscaled units for the box distribution of activation energies $[p(E)=1$, $0 \leqslant E \leqslant 1$ ] at four different $\beta$ 's. (b) The same data scaled according to Eq. (7), clearly converging to one single curve as $\beta \rightarrow \infty$. (c) Data for five different activation energy probability distributions at large $\beta$ 's, showing universality. The solid curve is the prediction of the effective-medium approximation (EMA) universality equation, Eq. (13). As a guide to the eye dots are shown marking a line with slope one. 
important in the next section.

Figure 8 shows results from computer simulations ofthe symmetric hopping model. Figure 8(a) gives the ac conductivity as a function of frequency in nonscaled units for the box distribution of activation energies at different temperatures. Figure 8(b) shows how these data, when scaled according to Eq. (7), converge to universality as $\beta \rightarrow \infty$. Finally, Fig. 8(c) shows the ac conductivities for five different activation energy probability distributions at large $\beta$, clearly showing universality. In Fig. 8(c) the solid line gives the effective-medium approximation universality prediction, which is not quite accurate. In two dimensions this approximation is even less accurate (Dyre, 1994).

The first hints of ac universality for hopping are found in an interesting paper by Summerfield (1985). He discussed several specific hopping models for electronic conduction in amorphous semiconductors and made the following discovery: When solved in the extended pair approximation (Summerfield and Butcher, 1982) all models have almost the same [scaled] ac conductivity. Numerical results for an energy-dependent hopping model implied similar behavior. He referred to this phenomenon as "quasi-universality." The quasi-universal ac conductivity was approximated by a constant plus a power law with exponent $n=0.725$.

\section{CAUSE OF UNIVERSALITY}

The macroscopic model and the symmetric hopping model both predict ac universality in the extreme disorder limit. The obvious question now is: What causes ac universality? Below, we present a physical picture of conduction in the extreme disorder limit and show how it implies ac universality. The scenario outlined builds on well-known insights gained during the last 30 years, but part of it is new and more speculative. The picture presented leads to two alternative analytic approximations to the universal ac conductivity, each applicable to either model.

\section{A. Role of percolation}

We shall argue that in both models ac universality arises because percolation controls the conductivity in the extreme disorder limit. Let us briefly recall what percolation is (Broadbent and Hammersley, 1957; Isichenko, 1992; Stauffer and Aharony, 1992). Consider a cubic lattice in any dimension and suppose each nearest-neighbor link is randomly marked with probability $p$. When $p$ is low few links are marked and connected clusters of marked links are small. Increasing $p$ the average cluster size increases. At the so-called percolation threshold $p_{c}$ an infinite cluster appears, the "percolation cluster." In two dimensions $p_{c}=0.5$ exactly, in three dimensions $p_{c} \cong 0.2488$ (Isichenko, 1992).

The percolation phenomenon is illustrated in Fig. 9. Figure 9(a) shows the situation below the threshold: It is not possible to follow the lines from one end of the figure to the other, so there is no infinite connected cluster
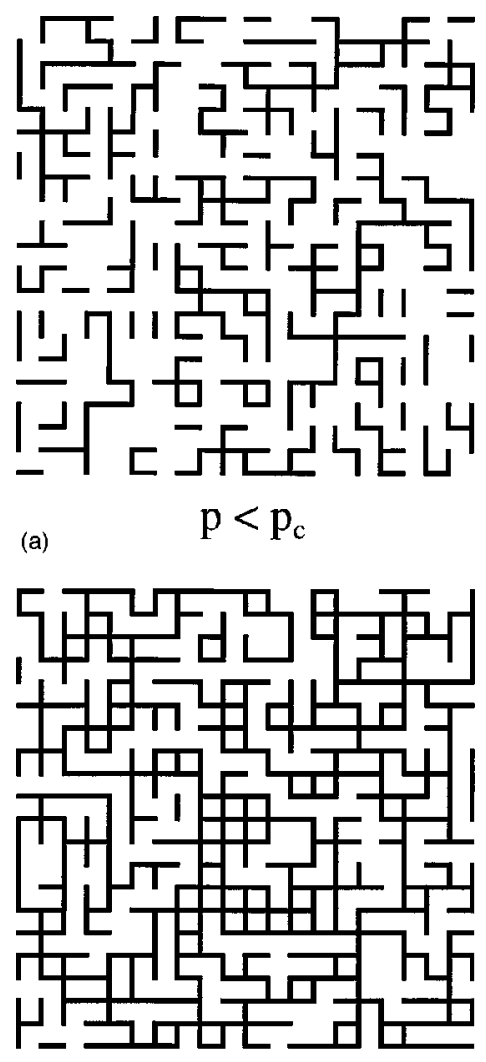

(b)

$$
\mathrm{p}>\mathrm{p}_{\mathrm{c}}
$$

FIG. 9. Percolation in two dimensions. On an underlying square lattice (not shown) each nearest-neighbor link is marked black with probability $p$. (a) shows the case when $p$ $=0.4$, which is below the percolation threshold $\left(p_{c}=0.5\right.$ in two dimensions). It is not possible to follow marked links across the entire figure. In (b) $p=0.6$ which is above the percolation threshold. This is reflected in the fact that it is now possible to find paths of marked links connecting opposite sides of the figure. The set of marked links stretching to infinity is termed the "percolation cluster." In the text it is argued that the percolation phenomenon lies behind ac universality in both models.

of marked links (lines). Figure 9(b), on the other hand, is above the percolation threshold. Here it is possible to follow lines across the figure (check this yourself!).

We first show how the dc conductivity activation energy $E_{c}$, defined by $\sigma(0) \propto \exp \left(-\beta E_{c}\right)$ at large $\beta$, for both models is determined from percolation arguments. In the dc limit the macroscopic model is described by a simple resistor network (the capacitors in Fig. 4, of course, play no role at zero frequency). When $\beta$ is large the random resistors cover many decades. Imagine now the resistors marked in order of increasing resistance. Clearly, a dc current through marked resistors is possible only when the fraction of marked resistors exceeds $p_{c}$. When this happens, due to the large spread of resistors, marking more resistors does not significantly change the admittance of the set of marked resistors. This admittance is dominated by the largest resistors among those marked just when percolation is reached (the "bottlenecks"). In particular, the temperature de- 
pendence of the overall admittance is the same as that of the bottleneck admittances. The dc conductivity activation energy $E_{c}$ is consequently determined by

$$
\int_{0}^{E_{c}} p(E) d E=p_{c} .
$$

Equation (23) was first derived by the above physical arguments (Ambegaokar, Halperin, and Langer, 1971; Shklovskii and Efros, 1971), and later proved rigorously (Tyč and Halperin, 1989).

Equation (23) is valid also for the symmetric hopping model: The main contribution to the (zero-field) meansquare displacement comes from charge carriers utilizing the links with the largest jump rates. Optimal charge carriers jump preferably on the percolation cluster, defined by marking links in order of decreasing jump rate until percolation. The optimal charge carriers must every now and then overcome the largest barriers on the percolation cluster. These barriers act as bottlenecks, so $E_{c}$ of Eq. (23) - the energy of the largest barriersdetermines the rate of mean-square displacement. Via Einstein's equation $\left\langle\Delta x^{2}(t)\right\rangle=2 D t$ and the fluctuationdissipation theorem [Eq. (15), here used at zero frequency] we conclude that the activation energy of $\sigma(0)$ is given by Eq. (23) also for hopping.

Before proceeding to discuss the origin of ac universality we must consider in somewhat more detail where the dc current flows. The problem is that the percolation cluster is a fractal and as such has zero bulk dc conductivity (Bouchaud and Georges, 1990). Thus besides the percolation cluster a tiny extra fraction of resistors/jump rates must also be involved in carrying the dc current. We shall term this enlarged cluster the "fat percolation cluster." How large is it? Probably, it only involves adding extra links with activation energies a few $k_{B} T$ above $E_{c}$, because adding more links cannot change the conductivity significantly (since these conduct poorly). Note that in the extreme disorder limit $(\beta \rightarrow \infty)$ the fat percolation cluster converges to the exact percolation cluster and the proof that $E_{c}$ is given by Eq. (23) still holds.

Our main assumption is now that not only dc, but also ac conduction mainly takes place on the fat percolation cluster. We thus assume that ac contributions from finite isolated "islands" are unimportant in the extreme disorder limit. At this point we differ crucially from previous physical pictures relating ac conductivity to the underlying percolation (Zvyagin, 1980; Böttger and Bryksin, 1985; Hunt, 1995).

Starting by the macroscopic model, we ask: Why is conductivity frequency independent at low frequencies and what determines the onset of ac conduction? To answer these questions, recall that all capacitors in Fig. 4 are equal. At very low frequencies each capacitor has numerically smaller admittance than that of its partner resistor. If frequency is increased, an increasing number of capacitor admittances become numerically larger than the admittance of their resistor partner. Whenever this happens for a link (an $R C$ element) we term it "affected." The average resistor current changes only insignificantly as long as none of the affected links are on the fat percolation cluster (which carries almost all currents). When frequency is continuously increased, at some point links on the fat percolation cluster do become affected. The first of these are the bottlenecks, the links with the largest resistors. From there on, as frequency is further increased, more and more links on the fat percolation cluster are affected-the node potentials on the cluster change and so do the resistor currents: The conductivity becomes frequency dependent. The frequency marking onset of ac conduction is roughly proportional to the dc conductivity, because both are roughly proportional to the bottleneck admittance. This explains Eq. (6), the Barton-Nakajima-Namikawa relation's rough proportionality between dc conductivity and dielectric loss peak frequency (recall that the latter marks onset of ac conduction).

For a fixed range of frequencies around $\omega_{m}$ the resistors of the affected links on the fat percolation cluster cover a corresponding range on a logarithmic scale. As $\beta \rightarrow \infty$ these resistors involve only a narrow range of activation energies around $E_{c} . \widetilde{\sigma}$ is dimensionless and can therefore depend only on dimensionless variables. Quantities with energy in their dimension which $\widetilde{\sigma}$ might involve are $E_{c}, p\left(E_{c}\right)$, and $\beta$. A change of the zero of the energy scale simply results in multiplication of conductivity and scaled frequency by constants. This does not change $\widetilde{\sigma}(\widetilde{\omega})$ so $\widetilde{\sigma}$ cannot depend on $E_{c}$. Therefore $\widetilde{\sigma}$ can depend on $p\left(E_{c}\right)$ only via the following dimensionless quantity: $\widetilde{p}=p\left(E_{c}\right) / \beta$. As $\beta$ diverges $\widetilde{p} \rightarrow 0$ and we have universality: $\widetilde{\sigma}$ becomes independent of both temperature and activation energy probability distribution. The only assumptions needed for this argument to work properly are that $p(E)$ is smooth at $E_{c}$ and that $p\left(E_{c}\right)>0$. Thus our reasoning does not apply to discontinuous situations like that of a binary inhomogeneous solid (Clerc et al., 1990).

To understand ac universality for hopping we shall consider the equilibrium mean-square displacement of a charge carrier. First we note that in terms of this quantity the frequency-dependent diffusion constant is given (Scher and Lax, 1973) by ${ }^{10}$

$$
D(\omega)=-\frac{\omega^{2}}{2} \int_{0}^{\infty}\left\langle\Delta x^{2}(t)\right\rangle e^{-i \omega t} d t
$$

As is easy to show, a linear mean-square displacement implies a frequency-independent diffusion constant. At long times the mean-square displacement indeed is linear in time. Consequently, the diffusion constant is frequency independent at sufficiently low frequencies. ${ }^{11}$

When $\beta$ is large the dominant contribution to the mean-square displacement comes from random walks on the fat percolation cluster. As we have seen already,

\footnotetext{
${ }^{10} \mathrm{An}$ implicit convergence factor $e^{-\epsilon t}(\lim \epsilon \rightarrow 0)$ is understood in the integral.

${ }^{11}$ For given $\omega$ the integral Eq. (24) is dominated by the contributions of $\left\langle\Delta x^{2}(t)\right\rangle$ at times $t$ given by $\omega t \sim 1$ (Tauberian theorem).
} 
the links with smallest jump rate $\Gamma_{c}$ on this cluster (the bottlenecks) determine the rate of mean-square displacement at long times which in turn determines the dc conductivity. The bottlenecks, however, also determine the frequency marking onset of ac conduction, $\omega_{m}$ : For a random walk on the fat percolation cluster many bottlenecks are passed in time $t$ whenever $\Gamma_{c} t \gg 1$. At these long times the cluster "looks" homogeneous and the mean-square displacement is linear in time. Consequently, conductivity is frequency independent whenever $\omega \ll \Gamma_{c} .{ }^{11}$ The mean-square displacement becomes nonlinear in time roughly when $\Gamma_{c} t \sim 1$, corresponding to frequencies $\omega \sim \Gamma_{c}$, because on that time scale random walks on the fat percolation cluster are limited to take place between bottlenecks and the cluster looks inhomogeneous. Since $\sigma(0)$ and $\omega_{m}$ are thus both roughly proportional to $\Gamma_{c}$, we now understand the origin of the Barton-Nakajima-Namikawa rough proportionality, Eq. (6), also for the hopping model.

From here on we argue much as for the macroscopic model. In a fixed range of frequencies around $\omega_{m}$, whenever $\beta$ is large only links with activation energies close to $E_{c}$ are important. $\widetilde{\sigma}$ can depend only on $\widetilde{p}$ $=p\left(E_{c}\right) / \beta$ which goes to zero in the extreme disorder limit. This establishes ac universality for the symmetric hopping model. Again, the only necessary assumptions are that $p(E)$ is smooth at $E_{c}$ and that $p\left(E_{c}\right)>0$.

The term "universality" became part of the physics vocabulary in the 1970s with the renormalization group theory of critical phenomena, one of the major advances in theoretical physics after World War II (Wilson, 1983; Goldenfeld, 1992; Fisher, 1998). A second-order phase transition is characterized by a number of critical exponents. Universality refers to the fact that these exponents depend only on dimension and order-parameter symmetry, not on microscopic details. Ac universality is not associated with exact power laws. Despite this there is a connection to critical phenomena, because ac universality is caused by the underlying percolation, and percolation is a critical phenomenon [see, e.g., Isichenko (1992)].

Approaching any second-order phase transition there is a diverging correlation length. Is ac universality also associated with a diverging length? Consider first hopping and define $l$ such that $l^{2}$ is the mean-square displacement at $t=1 / \omega_{m}$. In the effective-medium approximation $l$ diverges as $\beta \rightarrow \infty$. This is confirmed by computer simulations (Schrøder, 1999). Presumably, $l$ is proportional to the correlation length of the fat percolation cluster (Bouchaud and Georges, 1990; Baskin et al., 1997). This identification makes it possible to define $l$ also for the macroscopic model, and even for this model associate ac universality with a diverging correlation length.

The existence of a length diverging as $\beta \rightarrow \infty$ implies that ac universality is probably robust to rather extensive modifications of the two models, for instance by allowing resistors/jump rates which are not uncorrelated from link to link. As long as the resistors/jump rates have a finite correlation length we expect there is ac universality in the extreme disorder limit.

\section{B. Percolation based approximations}

To calculate the universal ac conductivity in either model the effective-medium approximation introduces a homogeneous effective medium. Although this approximation works well for the macroscopic model it is less successful for hopping. Is it possible to construct better approximations by making use of the above physical picture of conduction in the extreme disorder limit? We shall attempt to do this in two steps, each time developing an approximation applicable to both models. We argue simultaneously for both models. In the dc limit, in fact, the two models are mathematically equivalent, ${ }^{12}$ because in steady state the master equation (14) may be identified with Kirchhoff's current law if probability is identified with potential and jump rate with inverse resistance (Miller and Abrahams, 1960).

Computer simulations have shown that at extreme disorder the dc current follows almost one-dimensional paths (Brown and Esser, 1995). A naive approach is to regard these "conducting paths" as strictly onedimensional. For both models links with activation energies larger than $E_{c}$ are outside the fat percolation cluster and contribute little to the conductivity. Thus we arrive at the percolation path approximation (PPA): "The universal ac conductivity is equal to that of the extreme disorder limit of a one-dimensional model with a sharp upper cutoff in the activation energy probability distribution."

It is easy to apply this approximation to the macroscopic model, because the one-dimensional analog of Fig. 4 is exactly solvable. The percolation path approximation for the universal ac conductivity thus found (Dyre, 1993) is

$$
\widetilde{\sigma}=\frac{i \widetilde{\omega}}{\ln (1+i \widetilde{\omega})} .
$$

This mathematical expression (thin dashed line in Fig. 10) was first derived as the continuous time random walk approximation to a simple hopping model (Dyre, 1988). Equation (25) gives a slightly more pronounced frequency dependence of the conductivity than the solution of the effective-medium approximation universality equation, Eq. (13).

To apply the percolation path approximation to hopping, the ac conductivity in one dimension with a sharp activation energy cutoff must be calculated in the extreme disorder limit. This can only be done approximately. Dyre and Schrøder (1996) showed that the effective-medium approximation in one dimension represents simulations there very well and leads to the following equation:

\footnotetext{
${ }^{12}$ Note that this fact explains why Eq. (23) for the dc conductivity activation energy $E_{c}$ applies to both models.
} 


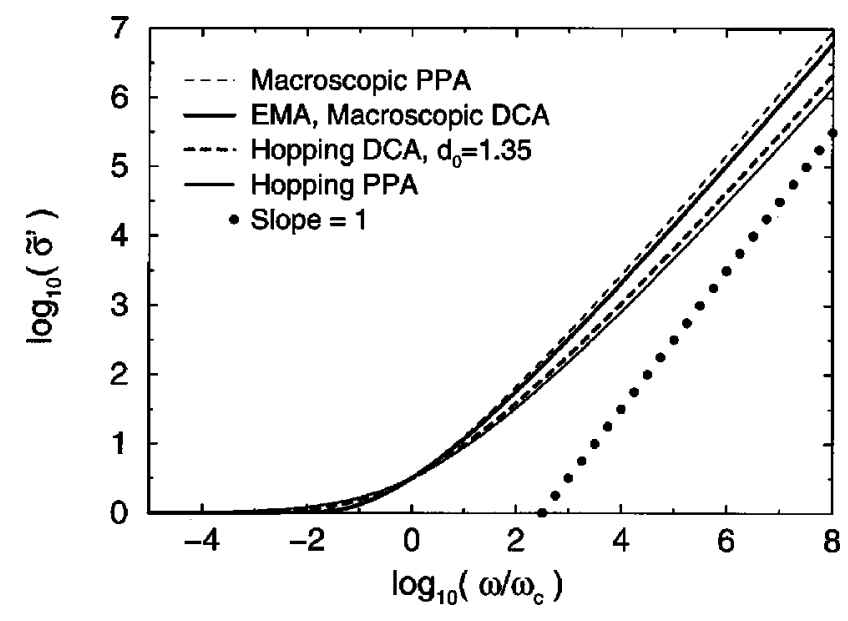

FIG. 10. Real parts of the four approximate analytical expressions for the universal ac conductivities of the two models (Table I) derived in: the effective-medium approximation (EMA), the percolation path approximation (PPA), and the diffusion cluster approximation (DCA). The frequency scaling is here defined such that all four curves coincide at $\log _{10}\left(\widetilde{\sigma}^{\prime}\right)$ $=0.5$. As a guide to the eye, dots are shown marking a line with slope one. All four expressions follow an approximate power law at high frequencies with an exponent which converges to one from below as frequency goes to infinity.

$$
\sqrt{\widetilde{\sigma}} \ln (1+\sqrt{i \widetilde{\omega} \widetilde{\sigma}})=\sqrt{i \widetilde{\omega}} .
$$

This is the percolation path approximation for hopping. Compared to the effective-medium approximation universality equation [Eq. (13)-applicable in two and more dimensions], Eq. (26) gives a somewhat less pronounced frequency dependence of the conductivity (thin solid line in Fig. 10).

The effective-medium approximation and the percolation path approximation are opposite extremes. The former views conduction as spatially homogeneous, the latter views it as strictly one-dimensional. In reality, conduction takes place on some complex subset of the fat percolation cluster which we term the diffusion cluster.

How to define the diffusion cluster? Consider the dc limit. Not all links on the fat percolation cluster carry current - there are dead ends. Removing these leaves us with the so-called backbone (Stauffer and Aharony, 1992). The backbone, which in ordinary threedimensional space has dimension 1.7, includes loops, however. Thus many pairs of sites are connected by two or more different paths on the backbone. In the extreme disorder limit one of these paths is by far most favorable. The backbone should therefore be further diluted by removing inefficient paths. A lower limit to this dilution is given by the set of "red bonds," those with the property that if one is removed the backbone is broken into two parts. The set of red bonds has dimension 1.1. This set is not connected, however, so the diffusion cluster must be larger. We thus expect for the diffusion cluster dimension $d_{0}$ :

$$
1.1<d_{0}<1.7 \text {. }
$$

To calculate $\widetilde{\sigma}$ in the diffusion cluster approximation (DCA), the effective-medium approximation is now applied to conduction on the diffusion cluster. For the macroscopic model this leads to Eq. (13), which applies in the extreme disorder limit whenever $d_{0}>1$ (Dyre, 1993). For hopping the situation is different. When $d_{0}$ $<2$ the hopping effective-medium approximation does not lead to Eq. (13). Instead, the following expression is arrived at (Schrøder and Dyre, 2000):

$$
\ln \widetilde{\sigma}=\left(\frac{i \widetilde{\omega}}{\widetilde{\sigma}}\right)^{d_{0} / 2} .
$$

A mathematically similar equation was derived by Zvyagin (1980) by arguments referring to percolation theory's finite cluster size statistics. The thick dashed line in Fig. 10 shows the solution of this equation for $d_{0}$ $=1.35$, the value which best fits hopping simulations (Schrøder and Dyre, 2000). All four approximate expressions for the universal ac conductivities of the two models are summarized in Table I.

Figure 11 compares the three approximations to hopping simulations, giving (Dyre and Schrøder, 1996) the apparent frequency exponent $n$ as a function of $\log _{10}\left(\widetilde{\sigma}^{\prime}\right)$. The diffusion cluster approximation gives the best fit to the numerical data. Recalling that the diffusion cluster approximation for the macroscopic model is equal to the effective-medium approximation universality equation, Eq. (13), which works well, our conclusion is simple: The diffusion cluster approximation works well for both models.

TABLE I. Ac universality equations.

\begin{tabular}{lcc}
\hline \hline & Macroscopic model & Symmetric hopping model \\
\hline Effective-medium approximation $^{\mathrm{a}}$ (EMA) & $\widetilde{\sigma} \ln \widetilde{\sigma}=i \widetilde{\omega}$ & $\widetilde{\sigma} \ln \widetilde{\sigma}=i \widetilde{\omega}$ \\
Percolation path approximation $^{\mathrm{b}}$ (PPA) & $\widetilde{\sigma}=\frac{i \widetilde{\omega}}{\ln (1+i \widetilde{\omega})}$ & $\sqrt{\widetilde{\sigma} \ln (1+\sqrt{i \widetilde{\omega} \widetilde{\sigma}}=\sqrt{i \widetilde{\omega}}}$ \\
Diffusion cluster approximation $^{\mathrm{c}}(\mathrm{DCA})$ & $\widetilde{\sigma} \ln \widetilde{\sigma}=i \widetilde{\omega}$ & $\ln \widetilde{\sigma}=\left(\frac{i \widetilde{\omega}}{\widetilde{\sigma}}\right)^{d_{0} / 2}$ \\
\hline \hline
\end{tabular}

${ }^{\mathrm{a}}$ Equation (13).

${ }^{\mathrm{b}}$ Equations (25) and (26).

${ }^{c}$ Equations (13) and (28). 


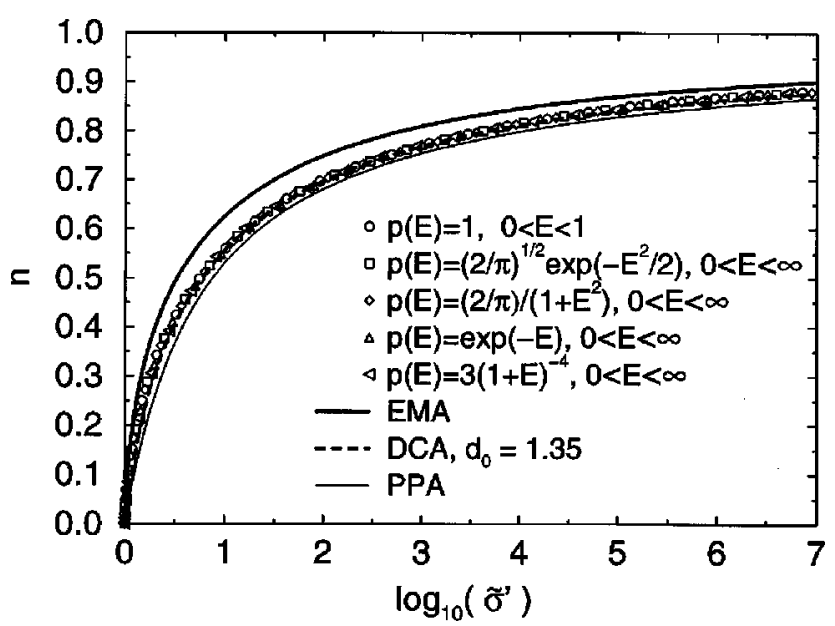

FIG. 11. The apparent power-law exponent $n \equiv d \ln \widetilde{\sigma}^{\prime} / d \ln \widetilde{\omega}$ plotted as a function of $\log _{10}\left(\widetilde{\sigma}^{\prime}\right)$ for the universal ac conductivity of the symmetric hopping model simulations [numerical data of Fig. 8(c)] [reproduced from Schrøder and Dyre (2000)]. The curves show the predictions of the effective medium approximation (EMA), the percolation path approximation (PPA), and the diffusion cluster approximation (DCA). The numerical data are best fitted by the diffusion cluster approximation with $d_{0}=1.35$ (dashed line, almost hidden by data points).

From a philosophical point of view one may wonder why the diffusion cluster approximation, which makes use of the effective-medium approximation, works better for hopping than the latter approximation itself. The reason is that the effective-medium approximation assumes an effective spatial homogeneity. This is done in a self-consistent manner to represent the actual inhomogeneity. But the fact remains that conduction in the extreme disorder limit is highly inhomogeneous. The diffusion cluster approximation takes this into account by assuming conduction effectively takes place in a lower dimension. This is the crucial idea. From there it is obvious to use the effective-medium approximation to find the ac conductivity in this lower dimension.

\section{DISCUSSION}

We have treated the two models on equal footing in order to focus on their common features. It should be emphasized, however, that while the symmetric hopping model and related models have been used extensively for many years, the macroscopic model was, and still is, less commonly used.

Before discussing model predictions and how they compare to experiment, let us briefly put things into a historical perspective. As mentioned in the Introduction, ac universality was generally recognized in the 1970s. Already in the 1950s, however, it was discovered that ion conducting oxide glasses have all more or less the same ac properties (Taylor 1956, 1957, 1959). At that time two models were proposed: (i) Stevels (1957) and Taylor $(1956,1959)$ assumed that ions jump between the minima of a random potential deriving from the random network structure of the glass (Zachariasen, 1932); (ii) Isard (1961) regarded the glass as a mixture of two phases with different conductivity. Little progress was made with either model, however, towards explaining ac universality. Note the close relationship between these early models and the two models discussed in this Colloquium.

For a long time it was believed that time-temperature superposition (the existence of a temperature-independent master curve) implies that any distribution of activation energies must be narrow compared to $k_{B} T$ (Taylor, 1959; Owen, 1963; Isard, 1970; Tomozawa, 1977). This belief was apparently confirmed by two further experimental facts: (i) The dc conductivity is not non-Arrhenius as naively expected if a broad range of activation energies is involved. (ii) The BartonNakajima-Namikawa rough proportionality $\sigma(0) \sim \omega_{m}$ [Eq. (6)] apparently implies that ac conduction is due to processes with the same activation energy as dc conduction. This is all wrong. Ironically, we now know that ac universality-and thereby time-temperature superposition-applies only when the range of activation energies is wide compared to $k_{B} T$. At the same time, everything appears to be controlled by just one activation energy, the $E_{c}$ of Eq. (23) identified by percolation theory.

\section{A. Model predictions}

Let us compare the assumptions of the two models. The symmetric hopping model assumes disorder on a microscopic scale, while the macroscopic model only assumes disorder on length scales large enough that a local conductivity may be defined. The symmetric hopping model ignores Coulomb interactions, while the macroscopic model takes these fully into account via Gauss' law. Finally, the electric field is assumed spatially homogenous in the hopping model whereas it varies throughout space in the macroscopic model [determined by Eq. (8)]. Despite these important differences, both models predict ac universality in the extreme disorder limit and the two universal ac conductivities are similar.

Three approximations to the universal ac conductivity of either model have been developed. Computer simulations show that for both models the best fit is provided by the diffusion cluster approximation [for the macroscopic model, of course, the effective-medium and the diffusion cluster approximations give the same universal $\widetilde{\sigma}(\widetilde{\omega})]$. The four analytical expressions derived (Fig. 10 and Table I) have the following in common: At low frequencies $(\widetilde{\omega} \ll 1)$ conductivity is frequency independent, at high frequencies $(\widetilde{\omega} \gg 1) \widetilde{\sigma}^{\prime}(\widetilde{\omega})$ follows an approximate power law with exponent $n<1$. It can be shown that for all four expressions the exponent is approximately given by

$$
\widetilde{\omega} \gg 1: \quad n=1-\frac{\alpha}{\ln \widetilde{\omega}} .
$$

One finds $\alpha=2$ for the effective-medium approximation, as well as for the macroscopic percolation path and the 
macroscopic diffusion cluster approximations. For the hopping percolation path approximation $\alpha=3$, while the hopping diffusion cluster approximation has $\alpha=1$ $+2 / d_{0}$. In all cases $n(\widetilde{\omega}) \rightarrow 1$ for $\widetilde{\omega} \rightarrow \infty$, which is also seen in computer simulations. This fact, in conjunction with the Barton-Nakajima-Namikawa relation's rough proportionality $\sigma(0) \sim \omega_{m}$ (also predicted by either mode $^{13}$ ) is the key to showing that both models predict all 16 points summarizing the experimental findings in Sec. III. The details of proving this (Dyre, 1988) are left to the reader.

We have focused exclusively on the real part of the ac conductivity, but the imaginary part also becomes universal in the extreme disorder limit and also follows an approximate power law above $\omega_{m}$ with an exponent ( $\neq n$, though) converging to 1 from below. There is, however, more to be said. Effective-medium calculations confirmed by computer simulations show that the imaginary part converges to universality more slowly than the real part as $\beta \rightarrow \infty$. The imaginary part therefore contains more system-specific information than the real part. $^{14}$

\section{B. Models versus experiment}

Figure 12 gives three examples comparing model predictions to experimental data. Figure 12(a) shows the very first published data indicating ac universality (Taylor, 1956, 1959) and simulations of hopping in the extreme disorder limit. In this figure we follow Taylor by presenting dielectric loss as a function of frequency. Figure 12(b) compares data for several sodium-germanate glasses (Sidebottom, 1999) to the same hopping model simulations. Given the fact that the symmetric hopping model in the extreme disorder limit has no fitting parameters, the fits in Figs. 12(a) and 12(b) are the best one can reasonably hope for. Figure 12(c) presents ac data which are better fitted by the macroscopic model. These data are for a metal cluster compound where conduction proceeds by electrons tunneling between metal islands. It is generally believed that these solids are well described by the symmetric hopping model (van Staveren, Brom, and de Jongh, 1991). We find, however, that the macroscopic model works better for these systems.

\footnotetext{
${ }^{13}$ Two of the four expressions in Table I (hopping percolation path approximation and hopping diffusion cluster approximation) have no dielectric loss peaks. These are artifacts of the approximations-in simulations both models do exhibit loss peaks. [In our hopping simulations we find that at extreme disorder the Barton-Nakajima-Namikawa relation, Eq. (5), is obeyed with $p=1.5 \pm 0.4$, while $p$ has not been determined for the macroscopic model.]

${ }^{14}$ In principle the imaginary part is uniquely determined from the real part via the Kramers-Kronig relation (Landau and Lifshitz, 1969), but this requires that the real part is known at all frequencies.
}

\section{Outlook}

There are many unsolved problems. Although one could argue - and we certainly do- that for both models ac universality in the extreme disorder limit has been demonstrated beyond any reasonable doubt, no rigorous proofs of ac universality exist.

Assuming ac universality, a number of problems remain:

- In regard to the diffusion cluster approximationthe one which works best for both models-it is important to precisely characterize the diffusion cluster and determine its dimension from analytical arguments and independent simulations.

- In both models there are links ( $R C$ elements/jump rates) with associated relaxation times much longer than the inverse loss peak frequency. For neither dc nor ac conductivity do these elements play a significant role. They have, however, other physical consequences like generating $1 / f$ noise of the dc conductivity (Morozovskii and Snarskii, 1993; Baskin et al., 1997). Is this confirmed by experiment? In other words: Do disordered solids have anomalously large $1 / f$ resistance fluctuations?

- In any hopping model which ignores charge carrier interactions the complete characterization of random walks lies in the $k$-dependent, frequency-dependent diffusion constant $D(k, \omega) .{ }^{15}$ Is $D(k, \omega)$ also universal in the extreme disorder limit? If yes, for which $k$ 's is this the case?

- As mentioned, the symmetric hopping model may be derived by (nonrigorously) linearizing the hopping model with random site energies and Fermi statistics. Does the latter more realistic model (Baranovskii and Cordes, 1999; Porto et al., 2000) exhibit ac universality in the extreme disorder limit and, if yes, does it have the same universal $\widetilde{\sigma}(\widetilde{\omega})$ as the symmetric hopping model?

- What about hopping models that are not symmetric: Do they exhibit ac universality always, sometimes, or never?

- Large-scale computer simulations of the macroscopic model are available in two dimensions (Dyre, 1993), but are still lacking in three dimensions. These simulations are needed, in particular, to confirm or reject the preliminary finding that the effective-medium approximation works well for this model also in three dimensions (Riedel and Dyre, 1994).

These problems all relate specifically to the two models. The overall purpose of future work, however, should be to advance our understanding to the point where reliable information about conduction mechanisms can be obtained from ac data and their deviations from universality. It seems to us that we are only at the beginning of such endeavors.

\footnotetext{
${ }^{15}$ If $P(\mathbf{r}, t)$ is the probability to find the charge carrier at site $\mathbf{r}$ at time $t$ given that it started at the origin at time 0 , $D(k, \omega)$ is defined as follows: $1 /\left[i \omega+k^{2} D(k, \omega)\right]$ $=\int_{0}^{\infty} d t e^{-i \omega t} \int d \mathbf{r} P(\mathbf{r}, t) e^{i \mathbf{k} \cdot \mathbf{r}}$. For small $k$ 's, $D(k, \omega)$ reduces to $D(\omega)$.
} 

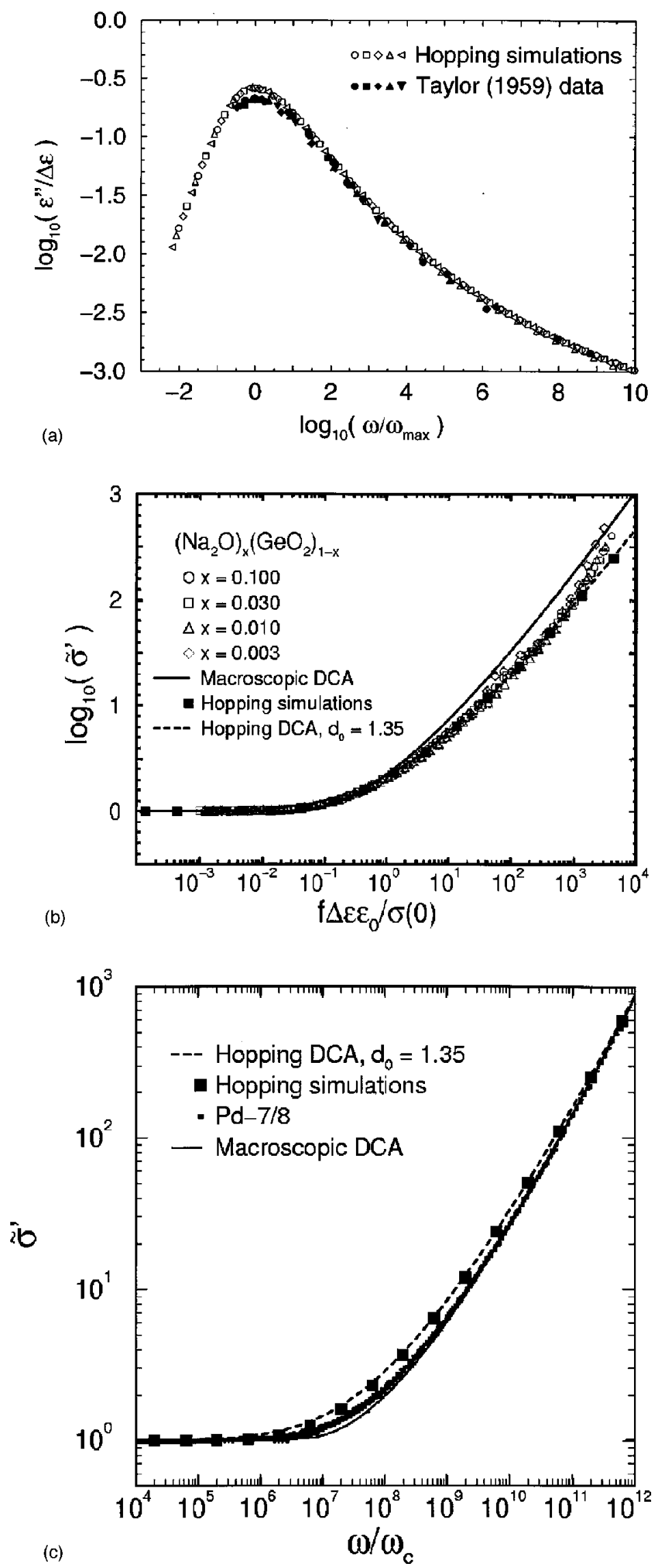

FIG. 12. Models versus experiment. (a) gives the dielectric loss for the first published data showing ac universality [solid symbols, data for five different ion conducting oxide glasses taken from Fig. 12 in Taylor (1959), an extension of Fig. 9 in Taylor (1956)]. The highfrequency data were obtained by Taylor by extrapolation, assuming time-temperature superposition. Taylor's data are compared to simulations of the symmetric hopping model in the extreme disorder limit [open symbols, as in Fig. 8(c)]. (b) shows the data of Sidebottom (1999) on sodium-germanate glasses compared to symmetric hopping model simulations in the extreme disorder limit, both data sets scaled according to Eq. (7) ( $f$ $=\omega / 2 \pi)$. Also shown are the best analytic approximations to the two models, the hopping diffusion cluster approximation (DCA) and the macroscopic diffusion cluster approximation (DCA) [reproduced from Schrøder and Dyre (2000)]. (c) shows data for the metal cluster compound Pd-7/8 (Reedijk et al., 1998) compared to symmetric hopping model simulations. The curves give the diffusion cluster approximations for hopping and for the macroscopic model. In this figure the frequency scaling was fixed by requiring same $\widetilde{\sigma}^{\prime}$ at the highest frequencies for data as well as for analytical approximations. 


\section{ACKNOWLEDGMENTS}

This paper is based on talks given by J.C.D. in Leipzig and Marburg (Germany) in May 1999. Friedrich Kremer and Sergei Baranovskii are thanked for their most kind hospitality in hosting these visits.

\section{REFERENCES}

Alexander, S., J. Bernasconi, W. R. Schneider, and R. Orbach, 1981, "Excitation dynamics in random one-dimensional systems," Rev. Mod. Phys. 53, 175-198.

Ambegaokar, V., B. I. Halperin, and J. S. Langer, 1971, “Hopping conductivity in disordered systems," Phys. Rev. B 4, 2612-2620.

Angell, C. A., 1990, "Dynamic processes in ionic glasses," Chem. Rev. 90, 523-542.

Balkan, N., P. N. Butcher, W. R. Hogg, A. R. Long, and S. Summerfield, 1985, "Analysis of frequency-dependent loss data in amorphous silicon and germanium," Philos. Mag. B 51, L7-L12.

Baranovskii, S. D., and H. Cordes, 1999, "On the conduction mechanism in ionic glasses," J. Chem. Phys. 111, 7546-7557.

Barton, J. L., 1966, "La relaxation diélectrique de quelques verres ternaires silice oxyde alcalin oxyde alcalino-terreux," Verres et Refr. 20, 328-334.

Baskin, E. M., A. A. Snarskii, A. Morozovskiy, J. Malinsky, and N. Abdellatif, 1997, "Effective properties for systems with distributed resistances in continuous space," Phys. Rev. B 56, 11611-11618.

Becker, R., 1967, Theory of Heat, second edition (Springer, Berlin).

Bergman, D. J., and D. Stroud, 1992, "Physical properties of macroscopically inhomogeneous media," in Solid State Physics, Vol. 46, edited by H. Ehrenreich and D. Turnbull (Academic, Boston), pp. 147-269.

Berryman, J. G., 1980, "Long-wavelength propagation in composite elastic media. I. Spherical inclusions," J. Acoust. Soc. Am. 68, 1809-1819.

Bianchi, R. F., P. H. Souza, T. J. Bonagamba, H. C. Panepucci, and R. M. Faria, 1999, "Ionic conduction and structural properties of organic-inorganic composite based on poly(propileneglycol)," Synth. Met. 102, 1186-1189.

Böttcher, C. J. F., and P. Bordewijk, 1978, Theory of Electric Polarization, Vol. 2, Dielectric Polarization (Elsevier, Amsterdam).

Böttger, H., and V. V. Bryksin, 1985, Hopping Conduction in Solids (Akademie, Berlin).

Bouchaud, J.-P., and A. Georges, 1990, “Anomalous diffusion in disordered media: Statistical mechanisms, models and physical applications," Phys. Rep. 195, 127-293.

Broadbent, S. R., and J. M. Hammersley, 1957, "Percolation processes. I. Crystals and mazes," Proc. Cambridge Philos. Soc. 53, 629-641.

Brom, H. B., J. A. Reedijk, H. C. F. Martens, L. J. Adriaanse, L. J. de Jongh, and M. A. J. Michels, 1998, "Frequency and temperature scaling in the conductivity and its structural consequences," Phys. Status Solidi B 205, 103-109.

Brown, R., and B. Esser, 1995, "Kinetic networks and orderstatistics for hopping in disordered systems," Philos. Mag. B 72, 125-148.

Bruggeman, D. A. G., 1935, "Berechnung verschiedener physikalischer Konstanten von heterogenen Substanzen. I. Diele- ktrizitätskonstanten und Leitfähigkeiten der Mischkörper aus isotropen Substanzen," Ann. Phys. (Leipzig) 24, 636-679.

Bryksin, V. V., 1980, "Frequency dependence of the hopping conductivity for three-dimensional systems in the framework of the effective-medium method," Fiz. Tverd. Tela (Leningrad) 22, 2441-2449 [Sov. Phys. Solid State 22, 1421-1426].

Butcher, P. N., 1974, "Stochastic interpretation of the rate equation formulation of hopping transport theory," J. Phys. C 7, 879-892.

Churchill, R. V., and J. W. Brown, 1984, Complex Variables and Applications, fourth edition (McGraw Hill, New York).

Clerc, J. P., G. Giraud, J. M. Laugier, and J. M. Luck, 1990, "The AC electrical conductivity of binary disordered systems, percolation clusters, fractals and related models," Adv. Phys. 39, 191-308.

Cramer, C., and M. Buscher, 1998, "Complete conductivity spectra of fast ion conducting silver iodide/silver selenate glasses," Solid State Ionics 105, 109-120.

Durand, B., G. Taillades, A. Pradel, M. Ribes, J. C. Badot, and N. Belhadj-Tahar, 1994, "Frequency dependence of conductivity in superionic conducting chalcogenide glasses," J. NonCryst. Solids 172-174, 1306-1314.

Dyre, J. C., 1988, "The random free energy barrier model for ac conduction in disordered solids," J. Appl. Phys. 64, 24562468.

Dyre, J. C., 1993, “Universal low-temperature ac conductivity of macroscopically disordered nonmetals," Phys. Rev. B 48, 12511-12526.

Dyre, J. C., 1994, "Studies of ac hopping conduction at low temperatures," Phys. Rev. B 49, 11709-11720; 50, 9692(E).

Dyre, J. C., 1995, "Energy master equation: A lowtemperature approximation to Bässler's random-walk model," Phys. Rev. B 51, 12276-12294.

Dyre, J. C., and J. M. Jacobsen, 1995, "Universal time dependence of the mean-square displacement in extremely rugged energy landscapes with equal minima," Phys. Rev. E 52, 2429-2433.

Dyre, J. C., and T. B. Schrøder, 1996, "Effective onedimensionality of universal ac hopping conduction in the extreme disorder limit," Phys. Rev. B 54, 14884-14887.

Economou, E. N., 1983, Green's Functions in Quantum Physics, second edition (Springer, Berlin).

Elliott, S. R., 1990, Physics of Amorphous Materials, second edition (Longman Scientific, London).

Elliott, S. R., 1994, "Frequency-dependent conductivity in ionically and electronically conducting amorphous solids," Solid State Ionics 70/71, 27-40.

Epstein, A. J., 1986, “AC conductivity of polyacetylene: Distinguishing mechanisms of charge transport," in Handbook of Conducting Polymers, Vol. 2, edited by T. A. Skotheim (Marcel Dekker, New York), pp. 1041-1097.

Fiegl, B., R. Kuhnert, M. Ben-Chorin, and F. Koch, 1994, "Evidence for grain boundary hopping transport in polycrystalline diamond films," Appl. Phys. Lett. 65, 371-373.

Fishchuk, I. I., 1986, "The AC conductivity and Hall effect in inhomogeneous semiconductors," Phys. Status Solidi A 93, 675-684.

Fisher, M. E., 1998, "Renormalization group theory: Its basis and formulation in statistical physics," Rev. Mod. Phys. 70, 653-681.

Funke, K., 1993, “Jump relaxation in solid electrolytes," Prog. Solid State Chem. 22, 111-195. 
Ghosh, A., and M. Sural, 1999, "A new scaling property of fluoride glasses: Concentration and temperature independence of the conductivity spectra," Europhys. Lett. 47, 688693.

Goldenfeld, N., 1992, Lectures on Phase Transitions and the Renormalization Group (Addison-Wesley, Reading, Massachusetts).

Haus, J. W., and K. W. Kehr, 1987, "Diffusion in regular and disordered lattices,” Phys. Rep. 150, 263-406.

Howell, F. S., R. A. Bose, P. B. Macedo, and C. T. Moynihan, 1974, "Electrical relaxation in a glass-forming molten salt," J. Phys. Chem. 78, 639-648.

Hughes, B. D., 1996, Random Walks and Random Environments, Vol. 2 (Clarendon, Oxford).

Hunt, A., 1995, "Approximate power-law conductivity in the multiple-hopping regime," J. Non-Cryst. Solids 183, 109-121. Isard, J. O., 1961, "A study of the migration loss in glass and a generalized method of calculating the rise of dielectric loss with temperature," Proc. Inst. Electr. Eng. 109B, Suppl. No. 22, 440-447 [The Institution of Electrical Engineers, Paper No. 3636].

Isard, J. O., 1970, "Dielectric dispersion in amorphous conductors," J. Non-Cryst. Solids 4, 357-365.

Isichenko, M. B., 1992, "Percolation, statistical topography, and transport in random media,' Rev. Mod. Phys. 64, 9611043.

Jastrzebska, M. M., S. Jussila, and H. Isotalo, 1998, “Dielectric response and a.c. conductivity of synthetic dopa-melanin polymer," J. Mater. Sci. 33, 4023-4028.

Jonscher, A. K., 1977, "The 'universal' dielectric response," Nature (London) 267, 673-679.

Jonscher, A. K., 1996, Universal Relaxation Law (Chelsea Dielectrics, London).

Kahnt, H., 1991, "Ionic transport in oxide glasses and frequency dependence of conductivity," Ber. Bunsenges. Phys. Chem. 95, 1021-1025.

Kimball, J. C., and L. W. Adams, Jr., 1978, "Hopping conduction and superionic conductors," Phys. Rev. B 18, 5851-5858.

Kirkpatrick, S., 1973, "Percolation and conduction," Rev. Mod. Phys. 45, 574-588.

Kittel, C., 1996, Introduction to Solid State Physics, seventh edition (Wiley, New York).

Kremer, F., 1999, private communication.

Kuanr, B. K., and G. P. Srivastava, 1994, "Dispersion observed in electrical properties of titanium-substituted lithium ferrites," J. Appl. Phys. 75, 6115-6117.

Kubo, R., 1957, "Statistical-mechanical theory of irreversible processes, I. General theory and simple applications to magnetic and conduction problems," J. Phys. Soc. Jpn. 12, 570586.

Kulkarni, A. R., P. Lunkenheimer, and A. Loidl, 1998, "Scaling behaviour in the frequency dependent conductivity of mixed alkali glasses," Solid State Ionics 112, 69-74.

Lakatos, A. I., and M. Abkowitz, 1971, "Electrical properties of amorphous $\mathrm{Se}, \mathrm{As}_{2} \mathrm{Se}_{3}$, and $\mathrm{As}_{2} \mathrm{~S}_{3}$," Phys. Rev. B 3, 17911800.

Landau, L. D., and E. M. Lifshitz, 1969, Statistical Physics, second edition (Pergamon, Oxford).

Landauer, R., 1978, "Electrical conductivity in inhomogeneous media," in Electrical Transport and Optical Properties of Inhomogeneous Media, AIP Conf. Proc. No. 40, edited by J. C. Garland and D. B. Tanner (AIP, New York), pp. 2-43.
Lidiard, A. B., 1957, "Ionic conductivity," in Handbuch der Physik, Vol. 20, edited by S. Flügge (Springer, Berlin), pp. 246-349.

Long, A. R., 1982, "Frequency-dependent loss in amorphous semiconductors," Adv. Phys. 31, 553-637.

Long, A. R., 1991, "Hopping conductivity in the intermediate frequency regime," in Hopping Transport in Solids, edited by M. Pollak and B. Shklovskii (Elsevier, Amsterdam), pp. 207231.

Long, A. R., and N. Balkan, 1980, "A.c. loss in amorphous germanium," J. Non-Cryst. Solids 35-36, 415-420.

Macdonald, J. R., 1997, "Possible universalities in the ac frequency response of dispersed, disordered materials," J. NonCryst. Solids 210, 70-86.

Mansingh, A., 1980, “A.c. conductivity of amorphous semiconductors," Bull. Mater. Sci. (India) 2, 325-351.

Maxwell, J. C., 1891, A Treatise on Electricity and Magnetism, Vol. 1, third edition (Clarendon, Oxford).

Miller, A., and E. Abrahams, 1960, "Impurity conduction at low concentrations," Phys. Rev. 120, 745-755.

Morozovskii, A. E., and A. A. Snarskii, 1993, "Percolation description of the conductivity of random networks with a broad spectrum of the distribution of resistances," Zh. Eksp. Teor. Fiz. 104, 4059-4071 [JETP 77, 959-965].

Mott, N. F., and E. A. Davis, 1979, Electronic Processes in Non-crystalline Materials, second edition (Clarendon, Oxford).

Nakajima, T., 1972, "Correlation between electrical conduction and dielectric polarization in inorganic glasses," in Conference on Electrical Insulation and Dielectric Phenomena (National Academy of Sciences, Washington, DC), pp. 168176.

Namikawa, H., 1975, "Characterization of the diffusion process in oxide glasses based on the correlation between electric conduction and dielectric relaxation," J. Non-Cryst. Solids 18, 173-195.

Ngai, K. L., and C. T. Moynihan, 1998, "The dynamics of mobile ions in ionically conducting glasses and other materials," MRS Bull. 23 (11), 51-56.

Owen, A. E., 1963, "Electric conduction and dielectric relaxation in glass," in Progress in Ceramic Science, Vol. 3, edited by J. E. Burke (Macmillan, New York), pp. 77-196.

Owen, A. E., 1977, "The electrical properties of glasses," J. Non-Cryst. Solids 25, 370-423.

Pollak, M., and T. H. Geballe, "Low-frequency conductivity due to hopping processes in silicon," 1961, Phys. Rev. 122, 1742-1753.

Porto, M., P. Maass, M. Meyer, A. Bunde, and W. Dieterich, 2000, "Hopping transport in the presence of site-energy disorder: Temperature and concentration scaling of conductivity spectra," Phys. Rev. B 61, 6057-6062.

Reedijk, J. A., L. J. Adriaanse, H. B. Brom, L. J. de Jongh, and G. Schmid, 1998, "Crossover from phonon- to photonmediated charge transport observed in metal-cluster compounds," Phys. Rev. B 57, R15116-R15119.

Rehwald, W., H. Kiess, and B. Binggeli, 1987, "Frequency dependent conductivity in polymers and other disordered materials," Z. Phys. B 68, 143-148.

Reitz, J. R., F. J. Milford, and R. W. Christy, 1993, Foundations of Electromagnetic Theory, fourth edition (AddisonWesley, Reading, Massachusetts). 
Riedel, T., and J. C. Dyre, 1994, "Low temperature universality in computer simulations of the macroscopic model for ac conduction in disordered solids," J. Non-Cryst. Solids 172174, 1419-1423.

Roling, B., 1998, "Scaling properties of the conductivity spectra of glasses and supercooled melts," Solid State Ionics 105, 185-193.

Roling, B., A. Happe, K. Funke, and M. D. Ingram, 1997, "Carrier concentration and relaxation spectroscopy: New information from scaling properties of conductivity spectra in ionically conducting glasses," Phys. Rev. Lett. 78, 2160-2163. Rozanski, S. A., F. Kremer, P. Köberle, and A. Laschewsky, 1995, "Relaxation and charge transport in mixtures of zwitterionic polymers and inorganic salts," Macromol. Chem. Phys. 196, 877-890.

Russell, B., 1946, A history of western philosophy (George Allen and Unwin Ltd, London).

Scher, H., and M. Lax, 1973, "Stochastic transport in a disordered solid. I. Theory; II. Impurity conduction," Phys. Rev. B 7, 4491-4502; 4502-4519.

Schrøder, T. B., 1999, "Hopping in disordered media: A model glass former and a hopping model," Ph.D. thesis (Roskilde University); e-print cond-mat/0005127.

Schrøder, T. B., and J. C. Dyre, 2000, "Scaling and universality of ac conduction in disordered solids," Phys. Rev. Lett. 84, 310-313.

Shklovskii, B. I., and A. L. Efros, 1971, "Impurity band and conductivity of compensated semiconductors," Zh. Éksp. Teor. Fiz. 60, 867-878 [Sov. Phys. JETP 33, 468-474].

Shklovskii, B. I., and A. L. Efros, 1984, Electronic Properties of Doped Semiconductors (Springer, Berlin).

Sidebottom, D. L., 1999, "Universal approach for scaling the ac conductivity in ionic glasses," Phys. Rev. Lett. 82, 36533656.

Stauffer, D., and A. Aharony, 1992, Introduction to Percolation Theory, second edition (Taylor and Francis, London).

Stein, D. L., and C. M. Newman, 1995, "Broken ergodicity and the geometry of rugged landscapes," Phys. Rev. E 51, 52285238.

Stevels, J. M., 1957, "The electrical properties of glass," in Handbuch der Physik, Vol. 20, edited by S. Flügge (Springer, Berlin), pp. 350-391.

Summerfield, S., 1985, "Universal low-frequency behaviour in the a.c. hopping conductivity of disordered systems," Philos. Mag. B 52, 9-22.
Summerfield, S., and P. N. Butcher, 1982, "A unified equivalent-circuit approach to the theory of AC and DC hopping conductivity in disordered systems," J. Phys. C: Solid State Phys. 15, 7003-7016.

Suzuki, M., 1980, “A.c. hopping conductivity in Mn-Co-Ni-Cu complex oxide semiconductors with spinel structure," J. Phys. Chem. Solids 41, 1253-1260.

Taylor, H. E., 1956, "The dielectric relaxation spectrum of glass," Trans. Faraday Soc. 52, 873-881.

Taylor, H. E., 1957, “An investigation of the dielectric relaxation exhibited by glass. Part I. Theory and methods of measurement," J. Soc. Glass Technol. 41, 350T-382T.

Taylor, H. E., 1959, "An investigation of the dielectric relaxation exhibited by glass. Part II. Soda-silica and soda-limesilica glasses," J. Soc. Glass Technol. 43, 124T-146T.

Tomozawa, M., 1977, "Dielectric characteristics of glass," in Treatise on Materials Science, Vol. 12, edited by M. Tomozawa (Academic, New York), pp. 283-345.

Tyč, S., and B. I. Halperin, 1989, "Random resistor network with an exponentially wide distribution of bond conductances," Phys. Rev. B 39, 877-880.

van Heumen, J., W. Wieczorek, M. Siekierski, and J. R. Stevens, 1995, "Conductivity and morphological studies of TPU-NH $\mathrm{CF}_{3} \mathrm{SO}_{3}$ polymeric electrolytes," J. Phys. Chem. 99, 15142-15152.

van Kampen, N. G., 1981, Stochastic Processes in Physics and Chemistry (North-Holland, Amsterdam).

van Staveren, M. P. J., H. B. Brom, and L. J. de Jongh, 1991, "Metal-cluster compounds and universal features of the hopping conductivity of solids," Phys. Rep. 208, 1-96.

Wagner, K. W., 1913, “Zur Theorie der unvollkommenen Dielektrika,’ Ann. Phys. (Leipzig) 40, 817-855.

Wilson, K. G., 1983, "The renormalization group and critical phenomena,” Rev. Mod. Phys. 55, 583-600.

Wolynes, P. G., 1992, "Randomness and complexity in chemical physics," Acc. Chem. Res. 25, 513-519.

Zachariasen, W. H., 1932, "The atomic arrangement in glass," J. Am. Chem. Soc. 54, 3841-3851.

Ziman, J. M., 1979, Models of Disorder (Cambridge University, Cambridge).

Zvyagin, I. P., 1980, "A percolative approach to the theory of the AC hopping conductivity," Phys. Status Solidi B 97, 143-149. 